\title{
Community Structure in Graphs
}

\author{
Santo Fortunato ${ }^{a}$, Claudio Castellano $^{b}$ \\ ${ }^{a}$ Complex Networks Lagrange Laboratory (CNLL), ISI Foundation, \\ Torino, Italy \\ ${ }^{b}$ SMC, INFM-CNR and Dipartimento di Fisica, "Sapienza" Uni- \\ versità di Roma, P. le A. Moro 2, 00185 Roma, Italy
}

\begin{abstract}
Graph vertices are often organized into groups that seem to live fairly independently of the rest of the graph, with which they share but a few edges, whereas the relationships between group members are stronger, as shown by the large number of mutual connections. Such groups of vertices, or communities, can be considered as independent compartments of a graph. Detecting communities is of great importance in sociology, biology and computer science, disciplines where systems are often represented as graphs. The task is very hard, though, both conceptually, due to the ambiguity in the definition of community and in the discrimination of different partitions and practically, because algorithms must find "good" partitions among an exponentially large number of them. Other complications are represented by the possible occurrence of hierarchies, i.e. communities which are nested inside larger communities, and by the existence of overlaps between communities, due to the presence of nodes belonging to more groups. All these aspects are dealt with in some detail and many methods are described, from traditional approaches used in computer science and sociology to recent techniques developed mostly within statistical physics.
\end{abstract}

\section{Introduction}

The origin of graph theory dates back to Euler's solution [1] of the puzzle of Königsberg's bridges in 1736. Since then a lot has been learned about graphs and their mathematical properties [2]. In the 20th century they have also become extremely useful as representation of a wide variety of systems in different areas. Biological, social, technological, and information networks can be studied as graphs, and graph analysis has become crucial to understand the features of these systems. For instance, social network analysis started in the 1930's and has become one of the most important topics in sociology 3, 4]. In recent times, the computer revolution has provided scholars with a huge amount of data and computational resources to process and analyse these data. The size of real networks one can potentially handle has also grown considerably, reaching 


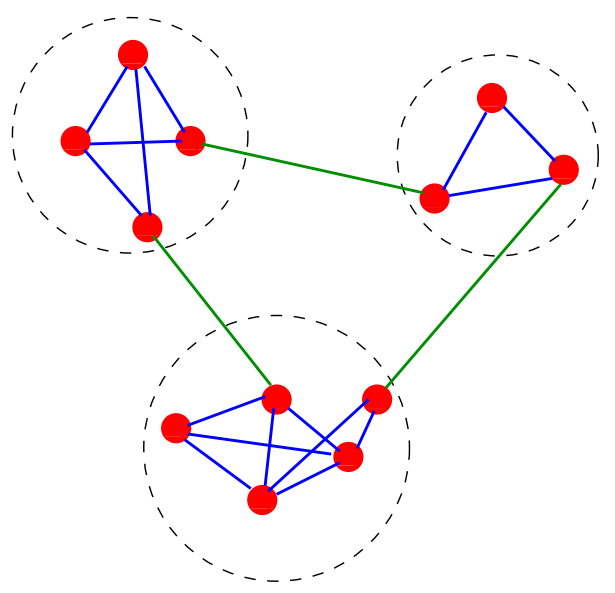

Figure 1: A simple graph with three communities, highlighted by the dashed circles.

millions or even billions of vertices. The need to deal with such a large number of units has produced a deep change in the way graphs are approached [5]- $[9]$.

Real networks are not random graphs. The random graph, introduced by P. Erdös and A. Rényi [10, is the paradigm of a disordered graph: in it, the probability of having an edge between a pair of vertices is equal for all possible pairs. In a random graph, the distribution of edges among the vertices is highly homogeneous. For instance, the distribution of the number of neighbours of a vertex, or degree, is binomial, so most vertices have equal or similar degree. In many real networks, instead, there are big inhomogeneities, revealing a high level of order and organization. The degree distribution is broad, with a tail that often follows a power law: therefore, many vertices with low degree coexist with some vertices with large degree. Furthermore, the distribution of edges is not only globally, but also locally inhomogeneous, with high concentrations of edges within special groups of nodes, and low concentrations between these groups. This feature of real networks is called community structure and is the topic of this chapter. In Fig. 1 a schematic example of a graph with community structure is shown.

Communities are groups of vertices which probably share common properties and/or play similar roles within the graph. So, communities may correspond to groups of pages of the World Wide Web dealing with related topics [11, to functional modules such as cycles and pathways in metabolic networks [12, 13, to groups of related individuals in social networks [14, 15, to compartments in food webs [16, 17, and so on.

Community detection is important for other reasons, too. Identifying modules and their boundaries allows for a classification of vertices, according to their topological position in the modules. So, vertices with a central position in their 
clusters, i.e. sharing a large number of edges with the other group partners, may have an important function of control and stability within the group; vertices lying at the boundaries between modules play an important role of mediation and lead the relationships and exchanges between different communities. Such classification seems to be meaningful in social [18,-20] and metabolic networks [12]. Finally, one can study the graph where vertices are the communities and edges are set between modules if there are connections between some of their vertices in the original graph and/or if the modules overlap. In this way one attains a coarse-grained description of the original graph, which unveils the relationships between modules. Recent studies indicate that networks of communities have a different degree distribution with respect to the full graphs [13; however, the origin of their structures can be explained by the same mechanism 21.

The aim of community detection in graphs is to identify the modules only based on the topology. The problem has a long tradition and it has appeared in various forms in several disciplines. For instance, in parallel computing it is crucial to know what is the best way to allocate tasks to processors so as to minimize the communications between them and enable a rapid performance of the calculation. This can be accomplished by splitting the computer cluster into groups with roughly the same number of processors, such that the number of physical connections between processors of different groups is minimal. The mathematical formalization of this problem is called graph partitioning. The first algorithms for graph partitioning were proposed in the early 1970's. Clustering analysis is also an important aspect in the study of social networks. The most popular techniques are hierarchical clustering and k-means clustering, where vertices are joined into groups according to their mutual similarity.

In a seminal paper, Girvan and Newman proposed a new algorithm, aiming at the identification of edges lying between communities and their successive removal, a procedure that after a few iterations leads to the isolation of modules [14. The intercommunity edges are detected according to the values of a centrality measure, the edge betweenness, that expresses the importance of the role of the edges in processes where signals are transmitted across the graph following paths of minimal length. The paper triggered a big activity in the field, and many new methods have been proposed in the last years. In particular, physicists entered the game, bringing in their tools and techniques: spin models, optimization, percolation, random walks, synchronization, etc., became ingredients of new original algorithms. Earlier reviews of the topic can be found in Refs. 22, 23.

Section 2 is about the basic elements of community detection, starting from the definition of community. The classical problem of graph partitioning and the methods for clustering analysis in sociology are presented in Sections 3 and 4 respectively. Section 5 is devoted to a description of the new methods. In Section 6 the problem of testing algorithms is discussed. Section[7introduces the description of graphs at the level of communities. Finally, Section 8 highlights the perspectives of the field and sorts out promising research directions for the future.

This chapter makes use of some basic concepts of graph theory, that can be 
found in any introductory textbook, like [2]. Some of them are briefly explained in the text.

\section{Elements of Community Detection}

The problem of community detection is, at first sight, intuitively clear. However, when one needs to formalize it in detail things are not so well defined. In the intuitive concept some ambiguities are hidden and there are often many equally legitimate ways of resolving them. Hence the term "Community Detection" actually indicates several rather different problems.

First of all, there is no unique way of translating into a precise prescription the intuitive idea of community. Many possibilities exist, as discussed below. Some of these possible definitions allow for vertices to belong to more than one community. It is then possible to look for overlapping or nonoverlapping communities. Another ambiguity has to do with the concept of community structure. It may be intended as a single partition of the graph or as a hierarchy of partitions, at different levels of coarse-graining. There is then a problem of comparison. Which one is the best partition (or the best hierarchy)? If one could, in principle, analyze all possible partitions of a graph, one would need a sensible way of measuring their "quality" to single out the partitions with the strongest community structure. It may even occur that one graph has no community structure and one should be able to realize it. Finding a good method for comparing partitions is not a trivial task and different choices are possible. Last but not least, the number of possible partitions grows faster than exponentially with the graph size, so that, in practice, it is not possible to analyze them all. Therefore one has to devise smart methods to find 'good' partitions in a reasonable time. Again, a very hard problem.

Before introducing the basic concepts and discussing the relevant questions it is important to stress that the identification of topological clusters is possible only if the graphs are sparse, i.e. if the number of edges $m$ is of the order of the number of nodes $n$ of the graph. If $m \gg n$, the distribution of edges among the nodes is too homogeneous for communities to make sense.

\subsection{Definition of Community}

The first and foremost problem is how to define precisely what a community is. The intuitive notion presented in the Introduction is related to the comparison of the number of edges joining vertices within a module ("intracommunity edges") with the number of edges joining vertices of different modules ("intercommunity edges"). A module is characterized by a larger density of links "inside" than "outside". This notion can be however formalized in many ways. Social network analysts have devised many definitions of subgroups with various degrees of internal cohesion among vertices [3, 4]. Many other definitions have been introduced by computer scientists and physicists. In general, the definitions can be classified in three main categories. 
- Local definitions. Here the attention is focused on the vertices of the subgraph under investigation and on its immediate neighbourhood, disregarding the rest of the graph. These prescriptions come mostly from social network analysis and can be further subdivided in self-referring, when one considers the subgraph alone, and comparative, when the mutual cohesion of the vertices of the subgraph is compared with their cohesion with the external neighbours. Self-referring definitions identify classes of subgraphs like cliques, n-cliques, $k$-plexes, etc.. They are maximal subgraphs, which cannot be enlarged with the addition of new vertices and edges without losing the property which defines them. The concept of clique is very important and often recurring when one studies graphs. A clique is a maximal subgraph where each vertex is adjacent to all the others. In the literature it is common to call cliques also non-maximal subgraphs. Triangles are the simplest cliques, and are frequent in real networks. Larger cliques are rare, so they are not good models of communities. Besides, finding cliques is computationally very demanding: the Bron-Kerbosch method 24] runs in a time growing exponentially with the size of the graph. The definition of clique is very strict. A softer constraint is represented by the concept of n-clique, which is a maximal subgraph such that the distance of each pair of its vertices is not larger than $n$. A k-plex is a maximal subgraph such that each vertex is adjacent to all the others except at most $k$ of them. In contrast, a $k$-core is a maximal subgraph where each vertex is adjacent to at least $k$ vertices within the subgraph. Comparative definitions include that of $L S$ set, or strong community, and that of weak community. An LS set is a subgraph where each node has more neighbours inside than outside the subgraph. Instead, in a weak community, the total degree of the nodes inside the community exceeds the external total degree, i.e. the number of links lying between the community and the rest of the graph. LS sets are also weak communities, but the inverse is not true, in general. The notion of weak community was introduced by Radicchi et al. 25].

- Global definitions. Communities are structural units of the graph, so it is reasonable to think that their distinctive features can be recognized if one analyses a subgraph with respect to the graph as a whole. Global definitions usually start from a null model, i.e. a graph which matches the original in some of its topological features, but which does not display community structure. After that, the linking properties of subgraphs of the initial graph are compared with those of the corresponding subgraphs in the null model. The simplest way to design a null model is to introduce randomness in the distribution of edges among the vertices. A random graph à la Erdös-Rényi, for instance, has no community structure, as any two vertices have the same probability to be adjacent, so there is no preferential linking involving special groups of vertices. The most popular null model is that proposed by Newman and Girvan and consists of a randomized version of the original graph, where edges are rewired at ran- 
dom, under the constraint that each vertex keeps its degree [26. This null model is the basic concept behind the definition of modularity, a function which evaluates the goodness of partitions of a graph into modules (see Section 2.2). Here a subset of vertices is a community if the number of edges inside the subset exceeds the expected number of internal edges that the subset would have in the null model. A more general definition, where one counts small connected subgraphs (motifs), and not necessarily edges, can be found in [27]. A general class of null models, including modularity, has been designed by Reichardt and Bornholdt [28].

- Definitions based on vertex similarity. In this last category, communities are groups of vertices which are similar to each other. A quantitative criterion is chosen to evaluate the similarity between each pair of vertices, connected or not. The criterion may be local or global: for instance one can estimate the distance between a pair of vertices. Similarities can be also extracted from eigenvector components of special matrices, which are usually close in value for vertices belonging to the same community. Similarity measures are at the basis of the method of hierarchical clustering, to be discussed in Section 4. The main problem in this case is the need to introduce an additional criterion to "close" the communities.

It is worth remarking that, in spite of the wide variety of definitions, in many detection algorithms communities are not defined at all, but are a byproduct of the procedure. This is the case of the divisive algorithms described in Section 5.1 and of the dynamic algorithms of Section 5.4 .

\subsection{Evaluating Partitions: Quality Functions}

Strictly speaking, a partition of a graph in communities is a split of the graph in clusters, with each vertex assigned to only one cluster. The latter condition may be relaxed, as shown in Section 2.4. Whatever the definition of community is, there is usually a large number of possible partitions. It is then necessary to establish which partitions exhibit a real community structure. For that, one needs a quality function, i.e. a quantitative criterion to evaluate how good a partition is. The most popular quality function is the modularity of Newman and Girvan [26]. It can be written in several ways, as

$$
Q=\frac{1}{2 m} \sum_{i j}\left(A_{i j}-\frac{k_{i} k_{j}}{2 m}\right) \delta\left(C_{i}, C_{j}\right),
$$

where the sum runs over all pairs of vertices, $A$ is the adjacency matrix, $k_{i}$ the degree of vertex $i$ and $m$ the total number of edges of the graph. The element $A_{i j}$ of the adjacency matrix is 1 if vertices $i$ and $j$ are connected, otherwise it is 0 . The $\delta$-function yields one if vertices $i$ and $j$ are in the same community, zero otherwise. Because of that, the only contributions to the sum come from vertex pairs belonging to the same cluster: by grouping them together the sum 
over the vertex pairs can be rewritten as a sum over the modules

$$
Q=\sum_{s=1}^{n_{m}}\left[\frac{l_{s}}{m}-\left(\frac{d_{s}}{2 m}\right)^{2}\right] .
$$

Here, $n_{m}$ is the number of modules, $l_{s}$ the total number of edges joining vertices of module $s$ and $d_{s}$ the sum of the degrees of the vertices of $s$. In Eq. 2 the first term of each summand is the fraction of edges of the graph inside the module, whereas the second term represents the expected fraction of edges that would be there if the graph were a random graph with the same degree for each vertex. In such a case, a vertex could be attached to any other vertex of the graph, and the probability of a connection between two vertices is proportional to the product of their degrees. So, for a vertex pair, the comparison between real and expected edges is expressed by the corresponding summand of Eq. 1 .

Eq. 2 embeds an implicit definition of community: a subgraph is a module if the number of edges inside it is larger than the expected number in modularity's null model. If this is the case, the vertices of the subgraph are more tightly connected than expected. Basically, if each summand in Eq. 2 is non-negative, the corresponding subgraph is a module. Besides, the larger the difference between real and expected edges, the more "modular" the subgraph. So, large positive values of $Q$ are expected to indicate good partitions. The modularity of the whole graph, taken as a single community, is zero, as the two terms of the only summand in this case are equal and opposite. Modularity is always smaller than one, and can be negative as well. For instance, the partition in which each vertex is a community is always negative. This is a nice feature of the measure, implying that, if there are no partitions with positive modularity, the graph has no community structure. On the contrary, the existence of partitions with large negative modularity values may hint to the existence of subgroups with very few internal edges and many edges lying between them (multipartite structure).

Modularity has been employed as quality function in many algorithms, like some of the divisive algorithms of Section [5.1. In addition, modularity optimization is itself a popular method for community detection (see Section 5.2). Modularity also allows to assess the stability of partitions [29] and to transform a graph into a smaller one by preserving its community structure [30].

However, there are some caveats on the use of the measure. The most important concerns the value of modularity for a partition. For which values one can say that there is a clear community structure in a graph? The question is tricky: if two graphs have the same type of modular structure, but different sizes, modularity will be larger for the larger graph. So, modularity values cannot be compared for different graphs. Moreover, one would expect that partitions of random graphs will have modularity values close to zero, as no community structure is expected there. Instead, it has been shown that partitions of random graphs may attain fairly large modularity values, as the probability that the distribution of edges on the vertices is locally inhomogeneous in specific realizations is not negligible [31. Finally, a recent analysis has proved that modularity increases if subgraphs smaller than a characteristic size are merged 32. This 


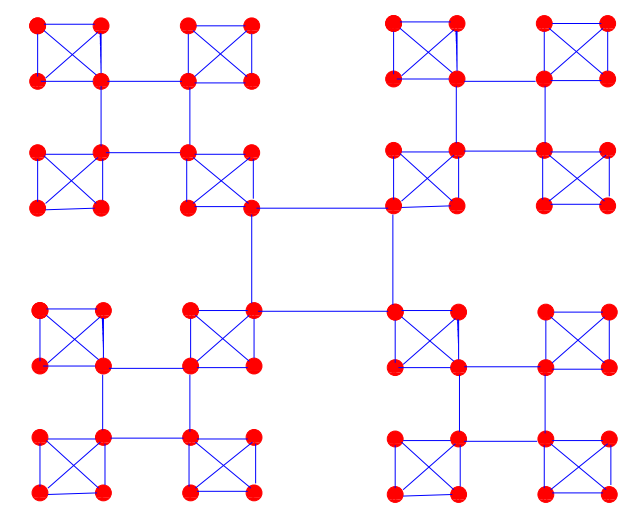

Figure 2: Schematic example of a hierarchical graph. Sixteen modules with four vertices each are clearly organized in groups of four.

fact represents a serious bias when one looks for communities via modularity optimization and is discussed in more detail in Section 5.2 .

\section{$2.3 \quad$ Hierarchies}

Graph vertices can have various levels of organization. Modules can display an internal community structure, i.e. they can contain smaller modules, which can in turn include other modules, and so on. In this case one says that the graph is hierarchical (see Fig. 2). For a clear classification of the vertices and their roles inside a graph, it is important to find all modules of the graph as well as their hierarchy.

A natural way to represent the hierarchical structure of a graph is to draw a dendrogram, like the one illustrated in Fig. 3. Here, partitions of a graph with twelve vertices are shown. At the bottom, each vertex is its own module. By moving upwards, groups of vertices are successively aggregated. Merges of communities are represented by horizontal lines. The uppermost level represents the whole graph as a single community. Cutting the diagram horizontally at some height, as shown in the figure (dashed line), displays one level of organization of the graph vertices. The diagram is hierarchical by construction: each community belonging to a level is fully included in a community at a higher level. Dendrograms are regularly used in sociology and biology. The technique of hierarchical clustering, described in Section 4, lends itself naturally to this kind of representation.

\subsection{Overlapping Communities}

As stated in Section 2.2, in a partition each vertex is generally attributed only to one module. However, vertices lying at the boundary between modules may be difficult to assign to one module or another, based on their connections with 


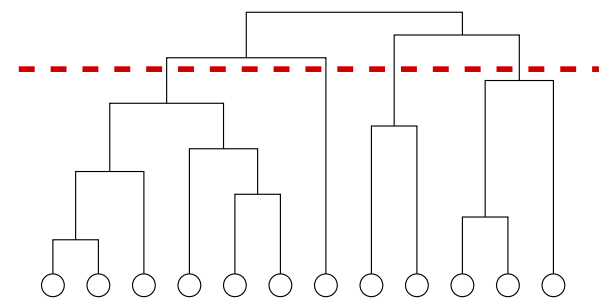

Figure 3: A dendrogram, or hierarchical tree. Horizontal cuts correspond to partitions of the graph in communities. Reprinted figure with permission from Newman MEJ, Girvan M, Physical Review E 69, 026113, 2004. Copyright 2004 by the Americal Physical Society.

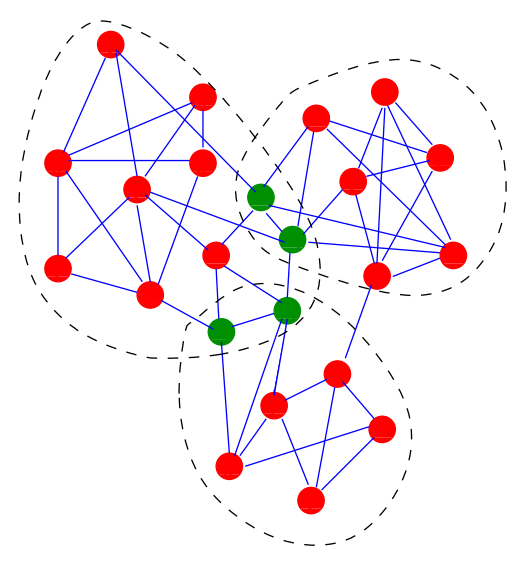

Figure 4: Overlapping communities. In the partition highlighted by the dashed contours, the green vertices are shared between more groups.

the other vertices. In this case, it makes sense to consider such intermediate vertices as belonging to more groups, which are then called overlapping communities (Fig. 4). Many real networks are characterized by a modular structure with sizeable overlaps between different clusters. In social networks, people usually belong to more communities, according to their personal life and interests: for instance a person may have tight relationships both with the people of its working environment and with other individuals involved in common free time activities.

Accounting for overlaps is also a way to better exploit the information that one can derive from topology. Ideally, one could estimate the degree of participation of a vertex in different communities, which corresponds to the likelihood that the vertex belongs to the various groups. Community detection algorithms, instead, often disagree in the classification of periferal vertices of modules, be- 


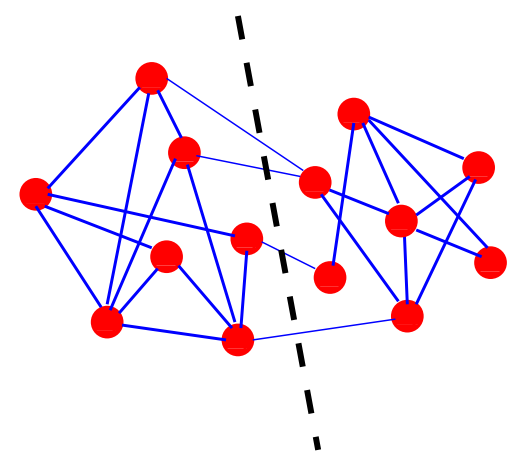

Figure 5: Graph partitioning. The cut shows the partition in two groups of equal size.

cause they are forced to put them in a single cluster, which may be the wrong one.

The problem of community detection is so hard that very few algorithms consider the possibility of having overlapping communities. An interesting method has been recently proposed by G. Palla et al. 13 and is described in Section 5.5 . For standard algorithms, the problem of identifying overlapping vertices could be addressed by checking for the stability of partitions against slight variations in the structure of the graph, as described in [33.

\section{Computer Science: Graph Partitioning}

The problem of graph partitioning consists in dividing the vertices in $g$ groups of predefined size, such that the number of edges lying between the groups is minimal. The number of edges running between modules is called cut size. Fig. 5 presents the solution of the problem for a graph with fourteen vertices, for $g=2$ and clusters of equal size.

The specification of the number of modules of the partition is necessary. If one simply imposed a partition with the minimal cut size, and left the number of modules free, the solution would be trivial, corresponding to all vertices ending up in the same module, as this would yield a vanishing cut size.

Graph partitioning is a fundamental issue in parallel computing, circuit partitioning and layout, and in the design of many serial algorithms, including techniques to solve partial differential equations and sparse linear systems of equations. Most variants of the graph partitioning problem are NP-hard, i.e. it is unlikely that the solution can be computed in a time growing as a power of the graph size. There are however several algorithms that can do a good job, even if their solutions are not necessarily optimal 34. Most algorithms perform a bisection of the graph, which is already a complex task. Partitions into more than two modules are usually attained by iterative bisectioning. 
The Kernighan-Lin algorithm [35] is one of the earliest methods proposed and is still frequently used, often in combination with other techniques. The authors were motivated by the problem of partitioning electronic circuits onto boards: the nodes contained in different boards need to be linked to each other with the least number of connections. The procedure is an optimization of a benefit function $Q$, which represents the difference between the number of edges inside the modules and the number of edges lying between them. The starting point is an initial partition of the graph in two clusters of the predefined size: such initial partition can be random or suggested by some information on the graph structure. Then, subsets consisting of equal numbers of vertices are swapped between the two groups, so that $Q$ has the maximal increase. To reduce the risk to be trapped in local maxima of $Q$, the procedure includes some swaps that decrease the function $Q$. After a series of swaps with positive and negative gains, the partition with the largest value of $Q$ is selected and used as starting point of a new series of iterations. The Kernighan-Lin algorithm is quite fast, scaling as $O\left(n^{2}\right)$ in worst-case time, $n$ being the number of vertices. The partitions found by the procedure are strongly dependent on the initial configuration and other algorithms can do better. However, the method is used to improve on the partitions found through other techniques, by using them as starting configurations for the algorithm.

Another popular technique is the spectral bisection method, which is based on the properties of the Laplacian matrix. The Laplacian matrix (or simply Laplacian) of a graph is obtained from the adjacency matrix $A$ by placing on the diagonal the degrees of the vertices and by changing the signs of the other elements. The Laplacian has all non-negative eigenvalues and at least one zero eigenvalue, as the sum of the elements of each row and column of the matrix is zero. If a graph is divided into $g$ connected components, the Laplacian would have $g$ degenerate eigenvectors with eigenvalue zero and can be written in block-diagonal form, i.e. the vertices can be ordered in such a way that the Laplacian displays $g$ square blocks along the diagonal, with entries different from zero, whereas all other elements vanish. Each block is the Laplacian of the corresponding subgraph, so it has the trivial eigenvector with components $(1,1,1, \ldots, 1,1)$. Therefore, there are $g$ degenerate eigenvectors with equal nonvanishing components in correspondence of the vertices of a block, whereas all other components are zero. In this way, from the components of the eigenvectors one can identify the connected components of the graph.

If the graph is connected, but consists of $g$ subgraphs which are weakly linked to each other, the spectrum will have one zero eigenvalue and $g-1$ eigenvalues which are close to zero. If the groups are two, the second lowest eigenvalue will be close to zero and the corresponding eigenvector, also called Fiedler vector, can be used to identify the two clusters as shown below.

Every partition of a graph with $n$ vertices in two groups can be represented by an index vector $\mathbf{s}$, whose component $\mathbf{s}_{i}$ is +1 if vertex $i$ is in one group and -1 if it is in the other group. The cut size $R$ of the partition of the graph in 
the two groups can be written as

$$
R=\frac{1}{4} \mathbf{s}^{T} \mathbf{L} \mathbf{s},
$$

where $\mathbf{L}$ is the Laplacian matrix and $\mathbf{s}^{T}$ the transpose of vector $\mathbf{s}$. Vector $\mathbf{s}$ can be written as $\mathbf{s}=\sum_{i} a_{i} \mathbf{v}_{i}$, where $\mathbf{v}_{i}, i=1, \ldots, n$ are the eigenvectors of the Laplacian. If $\mathbf{s}$ is properly normalized, then

$$
R=\sum_{i} a_{i}^{2} \lambda_{i},
$$

where $\lambda_{i}$ is the Laplacian eigenvalue corresponding to eigenvector $\mathbf{v}_{i}$. It is worth remarking that the sum contains at most $n-1$ terms, as the Laplacian has at least one zero eigenvalue. Minimizing $R$ equals to the minimization of the sum on the right-hand side of Eq. 4. This task is still very hard. However, if the second lowest eigenvector $\lambda_{2}$ is close enough to zero, a good approximation of the minimum can be attained by choosing $\mathbf{s}$ parallel to the Fiedler vector $\mathbf{v}_{2}$ : this would reduce the sum to $\lambda_{2}$, which is a small number. But the index vector cannot be perfectly parallel to $\mathbf{v}_{2}$ by construction, because all its components are equal in modulus, whereas the components of $\mathbf{v}_{2}$ are not. The best one can do is to match the signs of the components. So, one can set $\mathbf{s}_{i}=+1(-1)$ if $\mathbf{v}_{2}^{i}>0(<0)$. It may happen that the sizes of the two corresponding groups do not match the predefined sizes one wishes to have. In this case, if one aims at a split in $n_{1}$ and $n_{2}=n-n_{1}$ vertices, the best strategy is to order the components of the Fiedler vector from the lowest to the largest values and to put in one group the vertices corresponding to the first $n_{1}$ components from the top or the bottom, and the remaining vertices in the second group. If there is a discrepancy between $n_{1}$ and the number of positive or negative components of $\mathbf{v}_{2}$, this procedure yields two partitions: the better solution is the one that gives the smaller cut size.

The spectral bisection method is quite fast. The first eigenvectors of the Laplacian can be computed by using the Lanczos method [36], that scales as $m /\left(\lambda_{3}-\lambda_{2}\right)$, where $m$ is the number of edges of the graph. If the eigenvalues $\lambda_{2}$ and $\lambda_{3}$ are well separated, the running time of the algorithm is much shorter than the time required to calculate the complete set of eigenvectors, which scales as $O\left(n^{3}\right)$. The method gives in general good partitions, that can be further improved by applying the Kernighan-Lin algorithm.

Other methods for graph partitioning include level-structure partitioning, the geometric algorithm, multilevel algorithms, etc. A good description of these algorithms can be found in Ref. [34.

Graph partitioning algorithms are not good for community detection, because it is necessary to provide as input both the number of groups and their size, about which in principle one knows nothing. Instead, one would like an algorithm capable to produce this information in its output. Besides, using iterative bisectioning to split the graph in more pieces is not a reliable procedure. 


\section{Social Science: Hierarchical and K-Means Clus- tering}

In social network analysis, one partitions actors/vertices in clusters such that actors in the same cluster are more similar between themselves than actors of different clusters. The two most used techniques to perform clustering analysis in sociology are hierarchical clustering and $k$-means clustering.

The starting point of hierarchical clustering is the definition of a similarity measure between vertices. After a measure is chosen, one computes the similarity for each pair of vertices, no matter if they are connected or not. At the end of this process, one is left with a new $n \times n$ matrix $X$, the similarity matrix. Initially, there are $n$ groups, each containing one of the vertices. At each step, the two most similar groups are merged; the procedure continues until all vertices are in the same group.

There are different ways to define the similarity between groups out of the matrix $X$. In single linkage clustering, the similarity between two groups is the minimum element $x_{i j}$, with $i$ in one group and $j$ in the other. On the contrary, the maximum element $x_{i j}$ for vertices of different groups is used in the procedure of complete linkage clustering. In average linkage clustering one has to compute the average of the $x_{i j}$.

The procedure can be better illustrated by means of dendrograms, like the one in Fig. 3. One should note that hierarchical clustering does not deliver a single partition, but a set of partitions.

There are many possible ways to define a similarity measure for the vertices based on the topology of the network. A possibility is to define a distance between vertices, like

$$
x_{i j}=\sqrt{\sum_{k \neq i, j}\left(A_{i k}-A_{j k}\right)^{2}} .
$$

This is a dissimilarity measure, based on the concept of structural equivalence. Two vertices are structurally equivalent if they have the same neighbours, even if they are not adjacent themselves. If $i$ and $j$ are structurally equivalent, $x_{i j}=0$. Vertices with large degree and different neighbours are considered very "far" from each other. Another measure related to structural equivalence is the Pearson correlation between columns or rows of the adjacency matrix,

$$
x_{i j}=\frac{\sum_{k}\left(A_{i k}-\mu_{i}\right)\left(A_{j k}-\mu_{j}\right)}{n \sigma_{i} \sigma_{j}},
$$

where the averages $\mu_{i}=\left(\sum_{j} A_{i j}\right) / n$ and the variances $\sigma_{i}=\sum_{j}\left(A_{i j}-\mu_{i}\right)^{2}$.

An alternative measure is the number of edge- (or vertex-) independent paths between two vertices. Independent paths do not share any edge (vertex), and their number is related to the maximum flow that can be conveyed between the two vertices under the constraint that each edge can carry only one unit of flow (max-flow/min-cut theorem). Similarly, one could consider all paths running between two vertices. In this case, there is the problem that the total number 
of paths is infinite, but this can be avoided if one performs a weighted sum of the number of paths, where paths of length $l$ are weighted by the factor $\alpha^{l}$, with $\alpha<1$. So, the weights of long paths are exponentially suppressed and the sum converges.

Hierarchical clustering has the advantage that it does not require a preliminary knowledge on the number and size of the clusters. However, it does not provide a way to discriminate between the many partitions obtained by the procedure, and to choose that or those that better represent the community structure of the graph. Moreover, the results of the method depend on the specific similarity measure adopted. Finally, it does not correctly classify all vertices of a community, and in many cases some vertices are missed even if they have a central role in their clusters 22 .

Another popular clustering technique in sociology is k-means clustering [37. Here, the number of clusters is preassigned, say $k$. The vertices of the graph are embedded in a metric space, so that each vertex is a point and a distance measure is defined between pairs of points in the space. The distance is a measure of dissimilarity between vertices. The aim of the algorithm is to identify $k$ points in this space, or centroids, so that each vertex is associated to one centroid and the sum of the distances of all vertices from their respective centroids is minimal. To achieve this, one starts from an initial distribution of centroids such that they are as far as possible from each other. In the first iteration, each vertex is assigned to the nearest centroid. Next, the centers of mass of the $k$ clusters are estimated and become a new set of centroids, which allows for a new classification of the vertices, and so on. After a sufficient number of iterations, the positions of the centroids are stable, and the clusters do not change any more. The solution found is not necessarily optimal, as it strongly depends on the initial choice of the centroids. The result can be improved by performing more runs starting from different initial conditions.

The limitation of k-means clustering is the same as that of the graph partitioning algorithms: the number of clusters must be specified at the beginning, the method is not able to derive it. In addition, the embedding in a metric space can be natural for some graphs, but rather artificial for others.

\section{New Methods}

From the previous two sections it is clear that traditional approaches to derive graph partitions have serious limits. The most important problem is the need to provide the algorithms with information that one would like to derive from the algorithms themselves, like the number of clusters and their size. Even when these inputs are not necessary, like in hierarchical clustering, there is the question of estimating the goodness of the partitions, so that one can pick the best one. For these reasons, there has been a major effort in the last years to devise algorithms capable of extracting a complete information about the community structure of graphs. These methods can be grouped in different categories. 


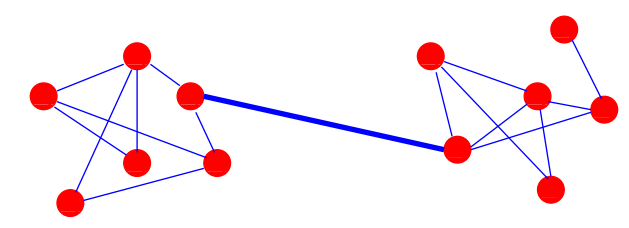

Figure 6: Edge betweenness is highest for edges connecting communities. In the figure, the thick edge in the middle has a much higher betweenness than all other edges, because all shortest paths connecting vertices of the two communities run through it.

\subsection{Divisive Algorithms}

A simple way to identify communities in a graph is to detect the edges that connect vertices of different communities and remove them, so that the clusters get disconnected from each other. This is the philosophy of divisive algorithms. The crucial point is to find a property of intercommunity edges that could allow for their identification. Any divisive method delivers many partitions, which are by construction hierarchical, so that they can be represented with dendrograms. Algorithm of Girvan and Newman. The most popular algorithm is that proposed by Girvan and Newman [14. The method is also historically important, because it marked the beginning of a new era in the field of community detection. Here edges are selected according to the values of measures of edge centrality, estimating the importance of edges according to some property or process running on the graph. The steps of the algorithm are:

1. Computation of the centrality for all edges;

2. Removal of edge with largest centrality;

3. Recalculation of centralities on the running graph;

4. Iteration of the cycle from step 2 .

Girvan and Newman focused on the concept of betweenness, which is a variable expressing the frequency of the participation of edges to a process. They considered three alternative definitions: edge betweenness, current-flow betweenness and random walk betweenness.

Edge betweenness is the number of shortest paths between all vertex pairs that run along the edge. It is an extension to edges of the concept of site betweenness, introduced by Freeman in 1977 [20. It is intuitive that intercommunity edges have a large value of the edge betweenness, because many shortest paths connecting vertices of different communities will pass through them (Fig. 6). The betweenness of all edges of the graph can be calculated in a time that scales as $O(m n)$, with techniques based on breadth-first-search [26, 38]. 
Current-flow betweenness is defined by considering the graph a resistor network, with edges having unit resistance. If a voltage difference is applied between any two vertices, each edge carries some amount of current, that can be calculated by solving Kirchoff's equations. The procedure is repeated for all possible vertex pairs: the current-flow betweenness of an edge is the average value of the current carried by the edge. Solving Kirchoff's equations requires the inversion of an $n \times n$ matrix, which can be done in a time $O\left(n^{3}\right)$ for a sparse matrix.

The random-walk betweenness of an edge says how frequently a random walker running on the graph goes across the edge. We remind that a random walker moving from a vertex follows each edge with equal probability. A pair of vertices is chosen at random, $s$ and $t$. The walker starts at $s$ and keeps moving until it hits $t$, where it stops. One computes the probability that each edge was crossed by the walker, and averages over all possible choices for the vertices $s$ and $t$. The complete calculation requires a time $O\left(n^{3}\right)$ on a sparse graph. It is possible to show that this measure is equivalent to current-flow betweenness [39].

Calculating edge betweenness is much faster than current-flow or random walk betweenness $\left(O\left(n^{2}\right)\right.$ versus $O\left(n^{3}\right)$ on sparse graphs). In addition, in practical applications the Girvan-Newman algorithm with edge betweenness gives better results than adopting the other centrality measures. Numerical studies show that the recalculation step 3 of Girvan-Newman algorithm is essential to detect meaningful communities. This introduces an additional factor $m$ in the running time of the algorithm: consequently, the edge betweenness version scales as $O\left(m^{2} n\right)$, or $O\left(n^{3}\right)$ on a sparse graph. Because of that, the algorithm is quite slow, and applicable to graphs with up to $n \sim 10000$ vertices, with current computational resources. In the original version of Girvan-Newman's algorithm [14, the authors had to deal with the whole hierarchy of partitions, as they had no procedure to say which partition is the best. In a successive refinement [26, they selected the partition with the largest value of modularity (see Section 2.2), a criterion that has been frequently used ever since. There have been countless applications of the Girvan-Newman method: the algorithm is now integrated in well known libraries of network analysis programs.

Algorithm of Tyler et al.. Tyler, Wilkinson and Huberman proposed a modification of the Girvan-Newman algorithm, to improve the speed of the calculation 40. The modification consists in calculating the contribution to edge betweenness only from a limited number of vertex pairs, chosen at random, deriving a sort of Monte Carlo estimate. The procedure induces statistical errors in the values of the edge betweenness. As a consequence, the partitions are in general different for different choices of the sampling pairs of vertices. However, the authors showed that, by repeating the calculation many times, the method gives good results, with a substantial gain of computer time. In practical examples, only vertices lying at the boundary between communities may not be clearly classified, and be assigned sometimes to a group, sometimes to another. The method has been applied to a network of people corresponding through email [40] and to networks of gene co-occurrences [41].

Algorithm of Fortunato et al.. An alternative measure of centrality for edges 
is information centrality. It is based on the concept of efficiency [42, which estimates how easily information travels on a graph according to the length of shortest paths between vertices. The information centrality of an edge is the variation of the efficiency of the graph if the edge is removed. In the algorithm by Fortunato, Latora and Marchiori 43, edges are removed according to decreasing values of information centrality. The method is analogous to that of Girvan and Newman, but slower, as it scales as $O\left(n^{4}\right)$ on a sparse graph. On the other hand, it gives a better classification of vertices when communities are fuzzy, i.e. with a high degree of interconnectedness.

Algorithm of Radicchi et al.. Because of the high density of edges within communities, it is easy to find loops in them, i.e. closed non-intersecting paths. On the contrary, edges lying between communities will hardly be part of loops. Based on this intuitive idea, Radicchi et al. proposed a new measure, the edge clustering coefficient, such that low values of the measure are likely to correspond to intercommunity edges [25]. The edge clustering coefficient generalizes to edges the notion of clustering coefficient introduced by Watts and Strogatz for vertices [44. The latter is the number of triangles including a vertex divided by the number of possible triangles that can be formed. The edge clustering coefficient is the number of loops of length $g$ including the edge divided by the number of possible cycles. Usually, loops of length $g=3$ or 4 are considered. At each iteration, the edge with smallest clustering coefficient is removed, the measure is recalculated again, and so on. The procedure stops when all clusters obtained are LS-sets or "weak" communities (see Section 2.1). Since the edge clustering coefficient is a local measure, involving at most an extended neighbourhood of the edge, it can be calculated very quickly. The running time of the algorithm to completion is $O\left(m^{4} / n^{2}\right)$, or $O\left(n^{2}\right)$ on a sparse graph, so it is much

shorter than the running time of the Girvan-Newman method. On the other hand, the method may give poor results when the graph has few loops, as it happens in several non-social networks. In this case, in fact, the edge clustering coefficient is small and fairly similar for all edges, and the algorithm may fail to identify the bridges between communities.

\subsection{Modularity Optimization}

If Newman-Girvan modularity $Q$ (Section 2.2) is a good indicator of the quality of partitions, the partition corresponding to its maximum value on a given graph should be the best, or at least a very good one. This is the main motivation for modularity maximization, perhaps the most popular class of methods to detect communities in graphs. An exhaustive optimization of $Q$ is impossible, due to the huge number of ways in which it is possible to partition a graph, even when the latter is small. Besides, the true maximum is out of reach, as it has been recently proved that modularity optimization is an NP-hard problem [45, so it is probably impossible to find the solution in a time growing polynomially with the size of the graph. However, there are currently several algorithms able to find fairly good approximations of the modularity maximum in a reasonable time. 
Greedy techniques. The first algorithm devised to maximize modularity was a greedy method of Newman [46. It is an agglomerative method, where groups of vertices are successively joined to form larger communities such that modularity increases after the merging. One starts from $n$ clusters, each containing a single vertex. Edges are not initially present, they are added one by one during the procedure. However, modularity is always calculated from the full topology of the graph, since one wants to find its partitions. Adding a first edge to the set of disconnected vertices reduces the number of groups from $n$ to $n-1$, so it delivers a new partition of the graph. The edge is chosen such that this partition gives the maximum increase of modularity with respect to the previous configuration. All other edges are added based on the same principle. If the insertion of an edge does not change the partition, i.e. the clusters are the same, modularity stays the same. The number of partitions found during the procedure is $n$, each with a different number of clusters, from $n$ to 1 . The largest value of modularity in this subset of partitions is the approximation of the modularity maximum given by the algorithm. The update of the modularity value at each iteration step can be performed in a time $O(n+m)$, so the algorithm runs to completion in a time $O((m+n) n)$, or $O\left(n^{2}\right)$ on a sparse graph, which is fast. In a later paper by Clauset et al. [47, it was shown that the calculation of modularity during the procedure can be performed much more quickly by use of max-heaps, special data structures created using a binary tree. By doing that, the algorithm scales as $O(m d \log n)$, where $d$ is the depth of the dendrogram describing the successive partitions found during the execution of the algorithm, which grows as $\log n$ for graphs with a strong hierarchical structure. For those graphs, the running time of the method is then $O\left(n \log ^{2} n\right)$, which allows to analyse the community structure of very large graphs, up to $10^{7}$ vertices. The greedy algorithm is currently the only algorithm that can be used to estimate the modularity maximum on such large graphs. On the other hand, the approximation it finds is not that good, as compared with other techniques. The accuracy of the algorithm can be considerably improved if one accounts for the size of the groups to be merged [48, or if the hierarchical agglomeration is started from some good intermediate configuration, rather than from the individual vertices [49].

Simulated annealing. Simulated annealing [50] is a probabilistic procedure for global optimization used in different fields and problems. It consists in performing an exploration of the space of possible states, looking for the global optimum of a function $F$, say its maximum. Transitions from one state to another occur with probability 1 if $F$ increases after the change, otherwise with a probability $\exp (\beta \Delta F)$, where $\Delta F$ is the decrease of the function and $\beta$ is an index of stochastic noise, a sort of inverse temperature, which increases after each iteration. The noise reduces the risk that the system gets trapped in local optima. At some stage, the system converges to a stable state, which can be an arbitrarily good approximation of the maximum of $F$, depending on how many states were explored and how slowly $\beta$ is varied. Simulated annealing was first employed for modularity optimization by R. Guimerá et al. 31]. Its standard implementation combines two types of "moves": local moves, where a single vertex is shifted from one cluster to another, taken at random; global 
moves, consisting of merges and splits of communities. In practical applications, one typically combines $n^{2}$ local moves with $n$ global ones in one iteration. The method can potentially come very close to the true modularity maximum, but it is slow. Therefore, it can be used for small graphs, with up to about $10^{4}$ vertices. Applications include studies of potential energy landscapes [51] and of metabolic networks [12].

Extremal optimization. Extremal optimization is a heuristic search procedure proposed by Boettcher and Percus [52, in order to achieve an accuracy comparable with simulated annealing, but with a substantial gain in computer time. It is based on the optimization of local variables, expressing the contribution of each unit of the system to the global function at study. This technique was used for modularity optimization by Duch and Arenas 53. Modularity can be indeed written as a sum over the vertices: the local modularity of a vertex is the value of the corresponding term in this sum. A fitness measure for each vertex is obtained by dividing the local modularity of the vertex by its degree. One starts from a random partition of the graph in two groups. At each iteration, the vertex with the lowest fitness is shifted to the other cluster. The move changes the partition, so the local fitnesses need to be recalculated. The process continues until the global modularity $Q$ cannot be improved any more by the procedure. At this stage, each cluster is considered as a graph on its own and the procedure is repeated, as long as $Q$ increases for the partitions found. The algorithm finds an excellent approximation of the modularity maximum in a time $O\left(n^{2} \log n\right)$, so it represents a good tradeoff between accuracy and speed. Spectral optimization. Modularity can be optimized using the eigenvalues and eigenvectors of a special matrix, the modularity matrix $B$, whose elements are

$$
B_{i j}=A_{i j}-\frac{k_{i} k_{j}}{2 m}
$$

where the notation is the same used in Eq. 11. The method [54, 55] is analogous to spectral bisection, described in Section 3. The difference is that here the Laplacian matrix is replaced by the modularity matrix. Between $Q$ and $B$ there is the same relation as between $R$ and $T$ in Eq. 3. so modularity can be written as a weighted sum of the eigenvalues of $B$, just like Eq. 4. Here one has to look for the eigenvector of $B$ with largest eigenvalue, $\mathbf{u}_{1}$, and group the vertices according to the signs of the components of $\mathbf{u}_{1}$, just like in Section 3. The Kernighan-Lin algorithm can then be used to improve the result. The procedure is repeated for each of the clusters separately, and the number of communities increases as long as modularity does. The advantage over spectral bisection is that it is not necessary to specify the size of the two groups, because it is determined by taking the partition with largest modularity. The drawback is similar as for spectral bisection, i.e. the algorithm gives the best results for bisections, whereas it is less accurate when the number of communities is larger than two. The situation could be improved by using the other eigenvectors with positive eigenvalues of the modularity matrix. In addition, the eigenvectors with the most negative eigenvalues are important to detect a possible multipartite structure of the graph, as they give the most relevant contribution to the 


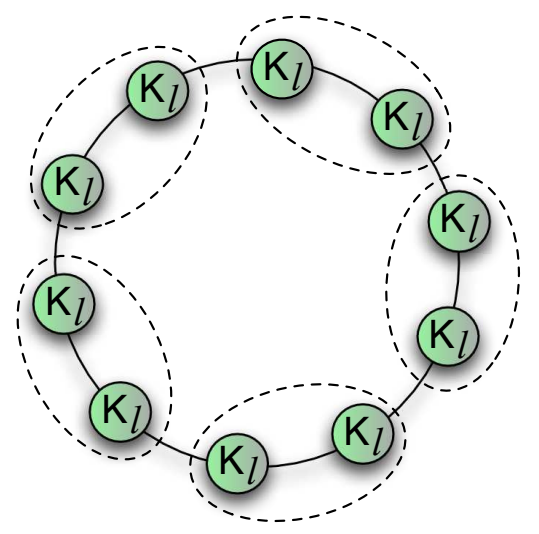

Figure 7: Resolution limit of modularity optimization. The natural community structure of the graph, represented by the individual cliques (circles), is not recognized by optimizing modularity, if the cliques are smaller than a scale depending on the size of the graph. Reprinted figure with permission from Fortunato S, Barthélemy M, Proceedings of the National Academy of Science of the USA, 104, 36 (2007). Copyright 2007 from the National Academy of Science of the USA.

modularity minimum. The algorithm typically runs in a time $O\left(n^{2} \log n\right)$ for a sparse graph, when one computes only the first eigenvector, so it is faster than extremal optimization, and slightly more accurate, especially for large graphs.

Finally, some general remarks on modularity optimization and its reliability. A large value for the modularity maximum does not necessarily mean that a graph has community structure. Random graphs can also have partitions with large modularity values, even though clusters are not explicitly built in [31, 56. Therefore, the modularity maximum of a graph reveals its community structure only if it is appreciably larger than the modularity maximum of random graphs of the same size 57 .

In addition, one assumes that the modularity maximum delivers the "best" partition of the network in communities. However, this is not always true [32]. In the definition of modularity (Eq. 2) the graph is compared with a random version of it, that keeps the degrees of its vertices. If groups of vertices in the graphs are more tightly connected than they would be in the randomized graph, modularity optimization would consider them as parts of the same module. But if the groups have less than $\sqrt{m}$ internal edges, the expected number of edges running between them in modularity's null model is less than one, and a single interconnecting edge would cause the merging of the two groups in the optimal partition. This holds for every density of edges inside the groups, even in the limit case in which all vertices of each group are connected to each other, i.e. if 
the groups are cliques. In Fig. [7 a graph is made out of $n_{c}$ identical cliques, with $l$ vertices each, connected by single edges. It is intuitive to think that the modules of the best partition are the single cliques: instead, if $n_{c}$ is larger than about $l^{2}$, modularity would be higher for the partition in which pairs of consecutive cliques are parts of the same module (indicated by the dashed lines in the figure). The problem holds for a wide class of possible null models [58. Attempts have been made to solve it within the modularity framework [59, 60, 61.

Modifications of the measure have also been suggested. Massen and Doye proposed a slight variation of modularity's null model [51]: it is still a graph with the same degree sequence as the original, and with edges rewired at random among the vertices, but one imposes the additional constraint that there can be neither multiple edges between a pair of vertices nor edges joining a vertex with itself (self-edges). Muff, Rao and Caflisch remarked that modularity's null model implicitly assumes that each vertex could be attached to any other, whether in real cases a cluster is usually connected to few other clusters 62. Therefore, they proposed a local version of modularity, in which the expected number of edges within a module is not calculated with respect to the full graph, but considering just a portion of it, namely the subgraph including the module and its neighbouring modules.

\subsection{Spectral Algorithms}

As discussed above, spectral properties of graph matrices are frequently used to find partitions. Traditional methods are in general unable to predict the number and size of the clusters, which instead must be fed into the procedure. Recent algorithms, reviewed below, are more powerful.

Algorithm of Donetti and Muñoz. An elegant method based on the eigenvectors of the Laplacian matrix has been devised by Donetti and Muñoz [63]. The idea is simple: the values of the eigenvector components are close for vertices in the same community, so one can use them as coordinates to represent vertices as points in a metric space. So, if one uses $M$ eigenvectors, one can embed the vertices in an $M$-dimensional space. Communities appear as groups of points well separated from each other, as illustrated in Fig. 8. The separation is the more visible, the larger the number of dimensions/eigenvectors $M$. The space points are grouped in communities by hierarchical clustering (see Section 4). The final partition is the one with largest modularity. For the similarity measure between vertices, Donetti and Muñoz used both the Euclidean distance and the angle distance. The angle distance between two points is the angle between the vectors going from the origin of the $M$-dimensional space to either point. Applications show that the best results are obtained with complete-linkage clustering. The algorithm runs to completion in a time $O\left(n^{3}\right)$, which is not fast. Moreover, the number $M$ of eigenvectors that are needed to have a clean separation of the clusters is not known a priori.

Algorithm of Capocci et al.. Similarly to Donetti and Muñoz, Capocci et al. used eigenvector components to identify communities 64. In this case the eigenvectors are those of the normal matrix, that is derived from the adjacency matrix 


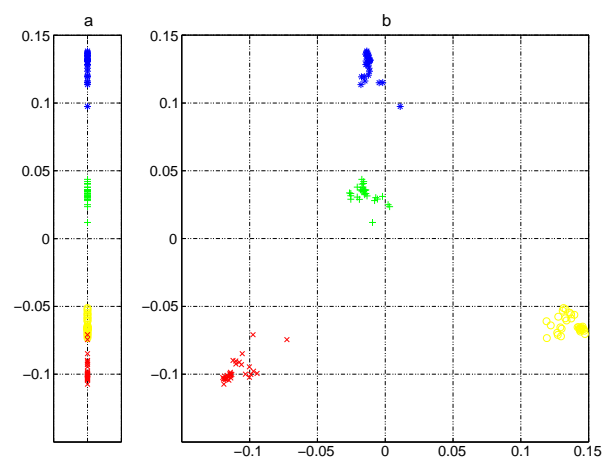

Figure 8: Spectral algorithm by Donetti and Muñoz. Vertex $i$ is represented by the values of the $i$ th components of Laplacian eigenvectors. In this example, the graph has an ad-hoc division in four communities, indicated by the colours. The communities are better separated in two dimensions (b) than in one (a). Reprinted figure with permission from Donetti L, Muñoz MA, Journal of Statistical Mechanics: Theory and Experiment, P10012 (2004). Copyright 2004 by the Institute of Physics.

by dividing each row by the sum of its elements. The eigenvectors can be quickly calculated by performing a constrained optimization of a suitable cost function. A similarity matrix is built by calculating the correlation between eigenvector components: the similarity between vertices $i$ and $j$ is the Pearson correlation coefficient between their corresponding eigenvector components, where the averages are taken over the set of eigenvectors used. The method can be extended to directed graphs. It is useful to estimate vertex similarities, however it does not provide a well-defined partition of the graph.

Algorithm of $W u$ and Huberman. A fast algorithm by Wu and Huberman identifies communities based on the properties of resistor networks [65. It is essentially a method for bisectioning graph, similar to spectral bisection, although partitions in an arbitrary number of communities can be obtained by iterative applications. The graph is transformed into a resistor network where each edge has unit resistance. A unit potential difference is set between two randomly chosen vertices. The idea is that, if there is a clear division in two communities of the graph, there will be a visible gap between voltage values for vertices at the borders between the clusters. The voltages are calculated by solving Kirchoff's equations: an exact resolution would be too time consuming, but it is possible to find a reasonably good approximation in a linear time for a sparse graph with a clear community structure, so the more time consuming part of the algorithm is the sorting of the voltage values, which takes time $O(n \log n)$. Any possible vertex pair can be chosen to set the initial potential difference, so the procedure should be repeated for all possible vertex pairs. The authors showed that this is not necessary, and that a limited number of sampling pairs 
is sufficient to get good results, so the algorithm scales as $O(n \log n)$ and is very fast. An interesting feature of the method is that it can quickly find the natural community of any vertex, without determining the complete partition of the graph. For that, one uses the vertex as source voltage and places the sink at an arbitrary vertex. The same feature is present in an older algorithm by Flake et al. [1], where one uses max-flow instead of current flow.

Previous works have shown that also the eigenvectors of the transfer matrix $\mathbf{T}$ can be used to extract useful information on community structure 66, 67. The element $T_{i j}$ of the transfer matrix is $1 / k_{j}$ if $i$ and $j$ are neighbours, where $k_{j}$ is the degree of $j$, otherwise it is zero. The transfer matrix rules the process of diffusion on graphs.

\subsection{Dynamic Algorithms}

This Section describes methods employing processes running on the graph, focusing on spin-spin interactions, random walk and synchronization.

Q-state Potts model. The Potts model is among the most popular models in statistical mechanics 68. It describes a system of spins that can be in $q$ different states. The interaction is ferromagnetic, i.e. it favours spin alignment, so at zero temperature all spins are in the same state. If antiferromagnetic interactions are also present, the ground state of the system may not be the one where all spins are aligned, but a state where different spin values coexist, in homogeneous clusters. If Potts spin variables are assigned to the vertices of a graph with community structure, and the interactions are between neighbouring spins, it is likely that the topological clusters could be recovered from like-valued spin clusters of the system, as there are many more interactions inside communities than outside. Based on this idea, inspired by an earlier paper by Blatt, Wiseman and Domany 69, Reichardt and Bornholdt proposed a method to detect communities that maps the graph onto a $q$-Potts model with nearest-neighbours interactions 70]. The Hamiltonian of the model, i.e. its energy, is the sum of two competing terms, one favoring spin alignment, one antialignment. The relative weight of these two terms is expressed by a parameter $\gamma$, which is usually set to the value of the density of edges of the graph. The goal is to find the ground state of the system, i.e. to minimize the energy. This can be done with simulated annealing [50, starting from a configuration where spins are randomly assigned to the vertices and the number of states $q$ is very high. The procedure is quite fast and the results do not depend on $q$. The method also allows to identify vertices shared between communities, from the comparison of partitions corresponding to global and local energy minima. More recently, Reichardt and Bornholdt derived a general framework 71, in which detecting community structure is equivalent to finding the ground state of a $q$-Potts model spin glass [72. Their previous method and modularity optimization are recovered as special cases. Overlapping communities can be discovered by comparing partitions with the same (minimal) energy, and hierarchical structure can be investigated by tuning a parameter acting on the density of edges of a reference graph without community structure. 
Random walk. Using random walks to find communities comes from the idea that a random walker spends a long time inside a community due to the high density of edges and consequent number of paths that could be followed. Zhou used random walks to define a distance between pairs of vertices [73: the distance between $i$ and $j$ is the average number of edges that a random walker has to cross to reach $j$ starting from $i$. Close vertices are likely to belong to the same community. The global attractor of a vertex $i$ is the closest vertex to $i$, whereas the local attractor of $i$ is its closest neighbour. Two types of communities are defined, according to local or global attractors: a vertex $i$ has to be put in the same community of its attractor and of all other vertices for which $i$ is an attractor. Communities must be minimal subgraphs, i.e. they cannot include smaller subgraphs which are communities according to the chosen criterion. Applications to real and artificial networks show that the method can find meaningful partitions. In a successive paper [74, Zhou introduced a measure of dissimilarity between vertices based on the distance defined above. The measure resembles the definition of distance based on structural equivalence of Eq. 5. where the elements of the adjacency matrix are replaced by the corresponding distances. Graph partitions are obtained with a divisive procedure that, starting from the graph as a single community, performs successive splits based on the criterion that vertices in the same cluster must be less dissimilar than a running threshold, which is decreased during the process. The hierarchy of partitions derived by the method is representative of actual community structures for several real and artificial graphs. In another work [75, Zhou and Lipowsky defined distances with biased random walkers, where the bias is due to the fact that walkers move preferentially towards vertices sharing a large number of neighbours with the starting vertex. A different distance measure between vertices based on random walks was introduced by Latapy and Pons [76. The distance is calculated from the probabilities that the random walker moves from a vertex to another in a fixed number of steps. Vertices are then grouped into communities through hierarchical clustering. The method is quite fast, running to completion in a time $O\left(n^{2} \log n\right)$ on a sparse graph.

Synchronization. Synchronization is another promising dynamic process to reveal communities in graphs. If oscillators are placed at the vertices, with initial random phases, and have nearest-neighbour interactions, oscillators in the same community synchronize first, whereas a full synchronization requires a longer time. So, if one follows the time evolution of the process, states with synchronized clusters of vertices can be quite stable and long-lived, so they can be easily recognized. This was first shown by Arenas, Díaz-Guilera and Pérez-Vicente 77. They used Kuramoto oscillators [78, which are coupled two-dimensional vectors endowed with a proper frequency of oscillations. If the interaction coupling exceeds a threshold, the dynamics leads to synchronization. Arenas et al. showed that the time evolution of the system reveals some intermediate time scales, corresponding to topological scales of the graph, i.e. to different levels of organization of the vertices. Hierarchical community structure can be revealed in this way (Fig. 9). Based on the same principle, Boccaletti et al. designed a community detection method based on synchronization [80]. 


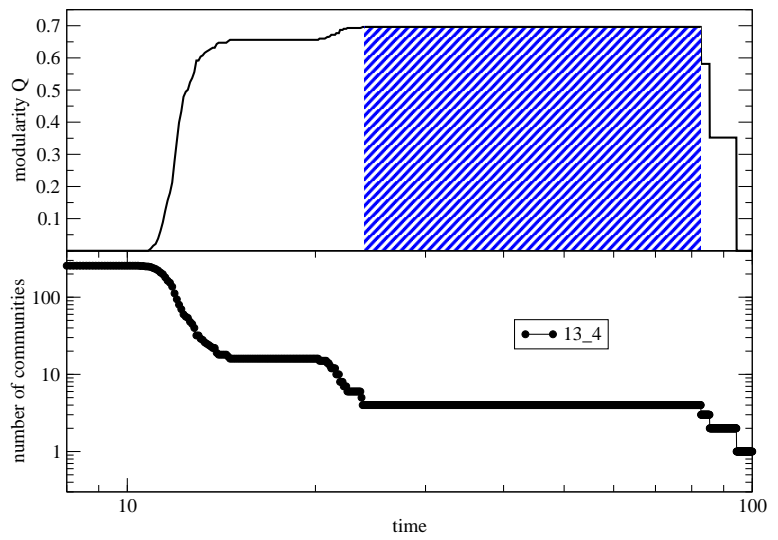

Figure 9: Number of clusters of synchronized Kuramoto oscillators as a function of time for a hierarchical graph. The two levels of community structure are revealed by the plateaus in the figure, which indicate the stability of those configurations. The top diagram shows the values of Newman-Girvan modularity $Q$ for the corresponding partitions. The shadowed area highlights the partition with largest modularity. Reprinted figure with permission from Arenas A, DíazGuilera A, European Physical Journal ST 143, 19 (2007). Copyright 2007 by EDP Sciences.

The synchronization dynamics is a variation of Kuramoto's model, the opinion changing rate (OCR) model [81. The evolution equations of the model are solved for decreasing values of a parameter that tunes the strength of the interaction coupling between neighbouring vertices. In this way, different partitions are recovered: the partition with the largest value of modularity is chosen. The algorithm scales in a time $O(m n)$, or $O\left(n^{2}\right)$ on sparse graphs, and gives good results on practical examples. However, synchronization-based algorithms may not be reliable when communities are very different in size.

\subsection{Clique Percolation}

In most of the approaches examined so far, communities have been characterized and discovered, directly or indirectly, by some global property of the graph, like betweenness, modularity, etc., or by some process that involves the graph as a whole, like random walks, synchronization, etc. But communities can be also interpreted as a form of local organization of the graph, so they could be defined from some property of the groups of vertices themselves, regardless of the rest of the graph. Moreover, very few of the algorithms presented so far 


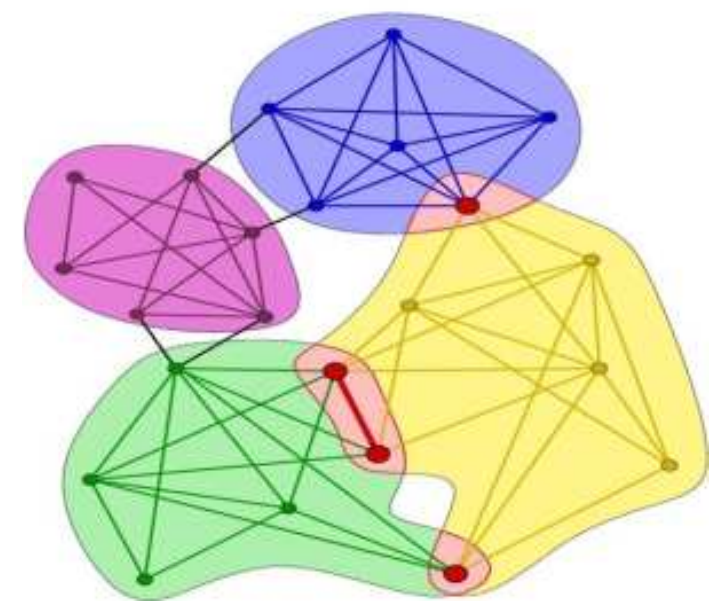

Figure 10: Clique Percolation Method. The example shows communities spanned by adjacent 3-cliques (triangles). Overlapping vertices are shown by the bigger dots. Reprinted figure with permission from Palla G, Derényi I, Farkas I and Vicsek T, Nature 435, 814 (2005). Copyright 2005 by the Nature Publishing Group.

are able to deal with the problem of overlapping communities (Section 2.4). A method that accounts both for the locality of the community definition and for the possibility of having overlapping communities is the Clique Percolation Method (CPM) by Palla et al. 13. It is based on the concept that the internal edges of community are likely to form cliques due to their high density. On the other hand, it is unlikely that intercommunity edges form cliques: this idea was already used in the divisive method of Radicchi et al. (see Section [5.1). Palla et al. define a $k$-clique as a complete graph with $k$ vertices. Notice that this definition is different from the definition of $n$-clique (see Section 2.1) used in social science. If it were possible for a clique to move on a graph, in some way, it would probably get trapped inside its original community, as it could not cross the bottleneck formed by the intercommunity edges. Palla et al. introduced a number of concepts to implement this idea. Two $k$-cliques are adjacent if they share $k-1$ vertices. The union of adjacent $k$-cliques is called $k$-clique chain. Two $k$-cliques are connected if they are part of a $k$ clique chain. Finally, a $k$-clique community is the largest connected subgraph obtained by the union of a $k$-clique and of all $k$-cliques which are connected to it. Examples of $k$-clique communities are shown in Fig. 10. One could say that a $k$-clique community is identified by making a $k$-clique "roll" over adjacent $k$-cliques, where rolling means rotating a $k$-clique about the $k-1$ vertices it shares with any adjacent $k$-clique. By construction, $k$-clique communities can share vertices, so they can be overlapping. There may be vertices belonging to 
non-adjacent $k$-cliques, which could be reached by different paths and end up in different clusters. In order to find $k$-clique communities, one searches first for maximal cliques, a task that is known to require a running time that grows exponentially with the size of the graph. However, the authors found that, for the real networks they analyzed, the procedure is quite fast, allowing to analyze graphs with up to $10^{5}$ vertices in a reasonably short time. The actual scalability of the algorithm depends on many factors, and cannot be expressed in closed form. The algorithm has been extended to the analysis of weighted 82 and directed [83] graphs. It was recently used to study the evolution of community structure in social networks 84]. A special software, called CFinder, based on the CPM, has been designed by Palla and coworkers and is freely available. The CPM has the same limit as the algorithm of Radicchi et al.: it assumes that the graph has a large number of cliques, so it may fail to give meaningful partitions for graphs with just a few cliques, like technological networks.

\subsection{Other Techniques}

This Section describes some algorithms that do not fit in the previous categories, although some overlap is possible.

Markov Cluster Algorithm (MCL). This method, invented by van Dongen [85, simulates a peculiar process of flow diffusion in a graph. One starts from the stochastic matrix of the graph, which is obtained from the adjacency matrix by dividing each element $A_{i j}$ by the degree of $i$. The element $S_{i j}$ of the stochastic matrix gives the probability that a random walker, sitting at vertex $i$, moves to $j$. The sum of the elements of each column of $S$ is one. Each iteration of the algorithm consists of two steps. In the first step, called expansion, the stochastic matrix of the graph is raised to an integer power $p$ (usually $p=2$ ). The entry $M_{i j}$ of the resulting matrix gives the probability that a random walker, starting from vertex $i$, reaches $j$ in $p$ steps (diffusion flow). The second step, which has no physical counterpart, consists in raising each single entry of the matrix $M$ to some power $\alpha$, where $\alpha$ is now real-valued. This operation, called inflation, enhances the weights between pairs of vertices with large values of the diffusion flow, which are likely to be in the same community. Next, the elements of each row must be divided by their sum, such that the sum of the elements of the row equals one and a new stochastic matrix is recovered. After some iterations, the process delivers a stable matrix, with some remarkable properties. Its elements are either zero or one, so it is a sort of adjacency matrix. Most importantly, the graph described by the matrix is disconnected, and its connected components are the communities of the original graph. The method is really simple to implement, which is the main reason of its success: as of now, the MCL is one of the most used clustering algorithms in bioinformatics. Due to the matrix multiplication of the expansion step, the algorithm should scale as $O\left(n^{3}\right)$, even if the graph is sparse, as the running matrix becomes quickly dense after a few steps of the algorithm. However, while computing the matrix multiplication, MCL keeps only a maximum number $k$ of non-zero elements per column, where $k$ is usually much smaller than $n$. So, the actual worst-case running time of the 
algorithm is $O\left(n k^{2}\right)$ on a sparse graph. A problem of the method is the fact that the final partition is sensitive to the parameter $\alpha$ used in the inflation step. Therefore several partitions can be obtained, and it is not clear which are the most meaningful or representative.

Maximum likelihood. Newman and Leicht have recently proposed an algorithm based on traditional tools and techniques of statistical inference [86. The method consists in deducing the group structure of the graph by checking which possible partition better "fits" the graph topology. The goodness of the fit is measured by the likelihood that the observed graph structure was generated by the particular set of relationships between vertices that define a partition. The latter is described by two sets of model parameters, expressing the size of the clusters and the connection preferences among the vertices, i.e. the probabilities that vertices of one cluster are linked to any vertex. The partition corresponding to the maximum likelihood is obtained by iterating a set of coupled equations for the variables, starting from a suitable set of initial conditions. Convergence is fast, so the algorithm could be applied to fairly large graphs, with up to about $10^{6}$ vertices. A nice feature of the method is that it discovers more general types of vertex classes than communities. For instance, multipartite structure could be uncovered, or mixed patterns where multipartite subgraphs coexist with communities, etc.. In this respect, it is more powerful than most methods of community detection, which are bound to focus only on proper communities, i.e. subgraphs with more internal than external edges. In addition, since partitions are defined by assigning probability values to the vertices, expressing the extent of their membership in a group, it is possible that some vertices are not clearly assigned to a group, but to more groups, so the method is able to deal with overlapping communities. The main drawback of the algorithm is the fact that one needs to specify the number of groups at the beginning of the calculation, a number that is often unknown for real networks. It is possible to derive this information self-consistently by maximizing the probability that the data are reproduced by partitions with a given number of clusters. But this procedure involves some degree of approximation, and the results are often not good.

L-shell method. This is an agglomerative method designed by Bagrow and Bollt [87. The algorithm finds the community of any vertex, although the authors also presented a more general procedure to identify the full community structure of the graph. Communities are defined locally, based on a simple criterion involving the number of edges inside and outside a group of vertices. One starts from a vertex-origin and keeps adding vertices lying on successive shells, where a shell is defined as a set of vertices at a fixed geodesic distance from the origin. The first shell includes the nearest neighbours of the origin, the second the next-to-nearest neighbours, and so on. At each iteration, one calculates the number of edges connecting vertices of the new layer to vertices inside and outside the running cluster. If the ratio of these two numbers ("emerging degree") exceeds some predefined threshold, the vertices of the new shell are added to the cluster, otherwise the process stops. Because of the local nature of the process, the algorithm is very fast and can identify communities very quickly. By 
repeating the process starting from every vertex, one could derive a membership matrix $M$ : the element $M_{i j}$ is one if vertex $j$ belongs to the community of vertex $i$, otherwise it is zero. The membership matrix can be rewritten by suitably permutating rows and columns based on their mutual distances. The distance between two rows (or columns) is defined as the number of entries whose elements differ. If the graph has a clear community structure, the membership matrix takes a block-diagonal form, where the blocks identify the communities. Unfortunately, the rearrangement of the matrix requires a time $O\left(n^{3}\right)$, so it is quite slow. In a different algorithm, local communities are discovered through greedy maximization of a local modularity measure 88 .

Algorithm of Eckmann and Moses. This is another method where communities are defined based on a local criterion 89. The idea is to use the clustering coefficient [44] of a vertex as a quantity to distinguish tightly connected groups of vertices. Many edges mean many loops inside a community, so the vertices of a community are likely to have a large clustering coefficient. The latter can be related to the average distance between pairs of neighbours of the vertex. The possible values of the distance are 1 (if neighbors are connected) or 2 (if they are not), so the average distance lies between 1 and 2 . The more triangles there are in the subgraph, the shorter the average distance. Since each vertex has always distance 1 from its neighbours, the fact that the average distance between its neighbours is different from 1 reminds what happens when one measures segments on a curved surface. Endowed with a metric, represented by the geodesic distance between vertices/points, and a curvature, the graph can be embedded in a geometric space. Communities appear as portions of the graph with a large curvature. The algorithm was applied to the graph representation of the World Wide Web, where vertices are Web pages and edges are the hyperlinks that take users from a page to the other. The authors found that communities correspond to Web pages dealing with the same topic.

Algorithm of Sales-Pardo et al.. This is an algorithm designed to detect hierarchical community structure (see Section 2.3), a realistic feature of many natural, social and technological networks, that most algorithms usually neglect. The authors [90] introduce first a similarity measure between pairs of vertices based on Newman-Girvan modularity: basically the similarity between two vertices is the frequency with which they coexist in the same community in partitions corresponding to local optima of modularity. The latter are configurations for which modularity is stable, i.e. it cannot increase if one shifts one vertex from one cluster to another or by merging or splitting clusters. Next, the similarity matrix is put in block-diagonal form, by minimizing a cost function expressing the average distance of connected vertices from the diagonal. The blocks correspond to the communities and the recovered partition represents the largest scale organization level. To determine levels at lower scales, one iterates the procedure for each subgraph identified at the previous level, which is considered as an independent graph. The method yields then a hierarchy by construction, as communities at each level are nested within communities at higher levels. The algorithm is not fast, as both the search of local optima for modularity and the rearrangement of the similarity matrix are performed with simulated anneal- 
ing, but delivers good results for computer generated networks, and meaningful partitions for some social, technological and biological networks.

Algorithm by Rosvall and Bergstrom. The modular structure can be considered as a reduced description of a graph to approximate the whole information contained in its adjacency matrix. Based on this idea, Rosvall and Bergstrom [91] envisioned a communication process in which a partition of a network in communities represents a synthesis $Y$ of the full structure that a signaler sends to a receiver, who tries to infer the original graph topology $X$ from it. The best partition corresponds to the signal $Y$ that contains the most information about $X$. This can be quantitatively assessed by the maximization of the mutual information $I(X ; Y)$ 92. The method is better than modularity optimization, especially when communities are of different size. The optimization of the mutual information is performed by simulated annealing, so the method is rather slow and can be applied to graphs with up to about $10^{4}$ vertices.

\section{Testing Methods}

When a community detection algorithm is designed, it is necessary to test its performance, and compare it with other methods. Ideally, one would like to have graphs with known community structure and check whether the algorithm is able to find it, or how closely can come to it. In any case, one needs to compare partitions found by the method with "real" partitions. How can different partitions of the same graph be compared? Danon et al. 93 used a measure borrowed from information theory, the normalized mutual information. One builds a confusion matrix $N$, whose element $N_{i j}$ is the number of vertices of the real community $i$ that are also in the detected community $j$. Since the partitions to be compared may have different numbers of clusters, $N$ is usually not a square matrix. The similarity of two partitions $A$ and $B$ is given by the following expression

$$
I(A, B)=\frac{-2 \sum_{i=1}^{c_{A}} \sum_{j=1}^{c_{B}} N_{i j} \log \left(N_{i j} N / N_{i .} N_{. j}\right)}{\sum_{i=1}^{c_{A}} N_{i .} \log \left(N_{i .} / N\right)+\sum_{j=1}^{c_{B}} N_{. j} \log \left(N_{. j} / N\right)},
$$

where $c_{B}\left(c_{A}\right)$ is the number of communities in partition $A(B), N_{i}$. is the sum of the elements of $N$ on row $i$ and $N_{. j}$ is the sum of the elements of $N$ on column $j$. Another useful measure of similarity between partitions is the Jaccard index, which is regularly used in scientometric research. Given two partitions $A$ and $B$, the Jaccard index is defined as

$$
I_{J}(A, B)=\frac{n_{11}}{n_{11}+n_{01}+n_{10}},
$$

where $n_{11}$ is the number of pairs of vertices which are in the same community in both partitions and $n_{01}\left(n_{10}\right)$ denotes the number of pairs of elements which are put in the same community in $A(B)$ and in different communities in $B(A)$. A nice presentation of criteria to compare partitions can be found in Ref. [94. 

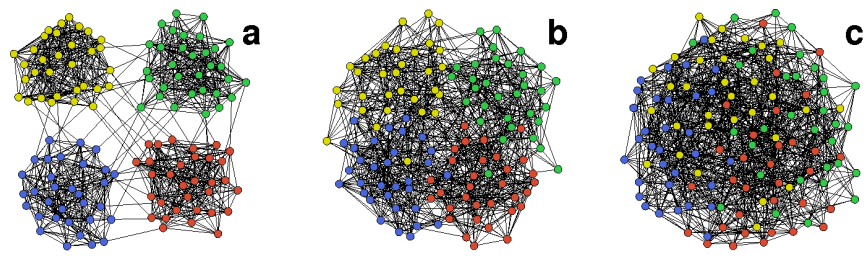

Figure 11: Benchmark of Girvan and Newman. The three pictures correspond to $z_{i n}=15$ (a), $z_{i n}=11$ (b) and $z_{i n}=8$ (c). In (c) the four groups are basically invisible. Reprinted figure with permission from Guimerà R, Amaral LAN, Nature 433, 895 (2005). Copyright 2005 by the Nature Publishing Group.

In the literature on community detection, algorithms have been generally tested on two types of graphs: computer generated graphs and real networks. The most famous computer generated benchmark is a class of graphs designed by Girvan and Newman [14. Each graph consists of 128 vertices, arranged in four groups with 32 vertices each: $1-32,33-64,65-96$ and $97-128$. The average degree of each vertex is set to 16 . The density of edges inside the groups is tuned by a parameter $z_{i n}$, expressing the average number of edges shared by each vertex of a group with the other members (internal degree). Naturally, when $z_{i n}$ is close to 16, there is a clear community structure (see Fig. 11), as most edges will join vertices of the same community, whereas when $z_{i n}<=8$ there are more edges connecting vertices of different communities and the graph looks fuzzy (see Fig. 117). In this way, one can realize different degrees of mixing between the groups. In this case the test consists in calculating the similarity between the partitions determined by the method at study and the natural partition of the graph in the four equal-sized groups. The similarity can be calculated by using the measure of Eq. 8, but in the literature one used a different quantity, i.e. the fraction of correctly classified vertices. A vertex is correctly classified if it is in the same cluster with at least 16 of its "natural" partners. If the model partition has clusters given by the merging of two or more natural groups, all vertices of the cluster are considered incorrectly classified. The number of correctly classified vertices is then divided by the total size of the graph, to yield a number between 0 and 1 . One usually builds many realizations of the graph for a particular value of $z_{i n}$ and computes the average fraction of correctly classified vertices, which is a measure of the sensitivity of the method. The procedure is then iterated for different values of $z_{i n}$. Many different algorithms have been compared with each other according to the diagram where the fraction of correctly classified vertices is plotted against $z_{i n}$. Most algorithms usually do a good job for large $z_{i n}$ and start to fail when $z_{i n}$ approaches 8 . The recipe to label vertices as correctly or incorrectly classified is somewhat arbitrary, though, and measures like those of Eqs. 8 and 9 are probably more objective. There is also a subtle problem concerning the reliability of the test. Because of the 


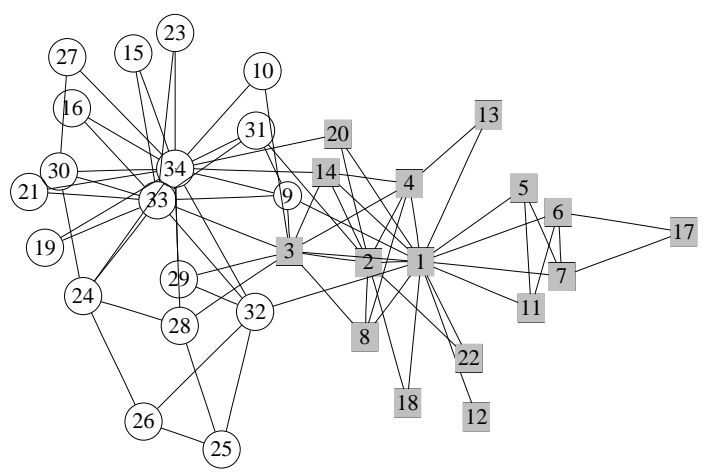

Figure 12: Zachary's karate club network, an example of graph with known community structure. Reprinted figure with permission from Newman MEJ, Girvan M, Physical Review E 69, 026113, 2004. Copyright 2004 by the Americal Physical Society.

randomness involved in the process of distributing edges among the vertices, it may well be that, in specific realizations of the graph, some vertices share more edges with members of another group than of their own. In this case, it is inappropriate to consider the initial partition in four groups as the real partition of the graph.

Tests on real networks usually focus on a limited number of examples, for which one has precise information about the vertices and their properties.

The most popular real network with a known community structure is the social network of Zachary's karate club (see Fig. 12). This is a social network representing the personal relationships between members of a karate club at an American university. During two years, the sociologist Wayne Zachary observed the ties between members, both inside and outside the club 95. At some point, a conflict arose between the club's administrator (vertex 1) and one of the teachers (vertex 33), which led to the split of the club in two smaller clubs, with some members staying with the administrator and the others following the instructor. Vertices of the two groups are highlighted by squares and circles in Fig. 12, The question is whether the actual social split could be predicted from the network topology. Several algorithms are actually able to identify the two classes, modulo a few intermediate vertices, which may be misclassified (e.g. vertices 3, 10). Other methods are less successful: for instance, the maximum of NewmanGirvan modularity corresponds to a split of the network in four groups [53, 63]. It is fundamental however to stress that the comparison of community structures detected by the various methods with the split of Zachary's karate club is based on a very strong assumption: that the split actually reproduced the separation of the social network in two communities. There is no real argument, beyond common wisdom, supporting this assumption.

Two other networks have frequently been used to test community detection 
algorithms: the network of American college football teams derived by Girvan and Newman [14] and the social network of bottlenose dolphins constructed by Lusseau [96]. Also for these networks the caveat applies: Nothing guarantees that "reasonable" communities, defined on the basis of non-topological information, must coincide with those detected by methods based only on topology.

\section{The Mesoscopic Description of a Graph}

Community detection algorithms have been applied to a huge variety of real systems, including social, biological and technological networks. The partitions found for each system are usually similar, as the algorithms, in spite of their specific implementations, are all inspired by close intuitive notions of community. What are the general properties of these partitions? The analysis of partitions and their properties delivers a mesoscopic description of the graph, where the communities, and not the vertices, are the elementary units of the topology. The term mesoscopic is used because the relevant scale here lies between the scale of the vertices and that of the full graph. A simple question is whether the communities of a graph are usually about of the same size or whether the community sizes have some special distribution. It turns out that the distribution of community sizes is skewed, with a tail that obeys a power law with exponents in the range between 1 and 3 [13, 22, 23, 47]. So, there seems to be no characteristic size for a community: small communities usually coexist with large ones. As an example, Fig. 13 shows the cumulative distribution of community sizes for a recommendation network of the online vendor Amazon.com. Vertices are products and there is a connection between item $A$ and $B$ is $B$ was frequently purchased by buyers of $A$. We remind that the cumulative distribution is the integral of the probability distribution: if the cumulative distribution is a power law with exponent $\alpha$, the probability distribution is also a power law with exponent $\alpha+1$.

If communities are overlapping, one could derive a network, where the communities are the vertices and pairs of vertices are connected if their corresponding communities overlap [13. Such networks seem to have some special properties. For instance, the degree distribution is a particular function, with an initial exponential decay followed by a slower power law decay. A recent analysis has shown that such distribution can be reproduced by assuming that the graph grows according to a simple preferential attachment mechanism, where communities with large degree have an enhanced chance to interact/overlap with new communities [21].

Finally, by knowing the community structure of a graph, it is possible to classify vertices according to their roles within their community, which may allow to infer individual properties of the vertices. A nice classification has been proposed by Guimerá and Amaral [12, 97. The role of a vertex depends on the values of two indices, the $z$-score and the participation ratio, that determine the position of the vertex within its own module and with respect to the other modules. The $z$-score compares the internal degree of the vertex in its module 


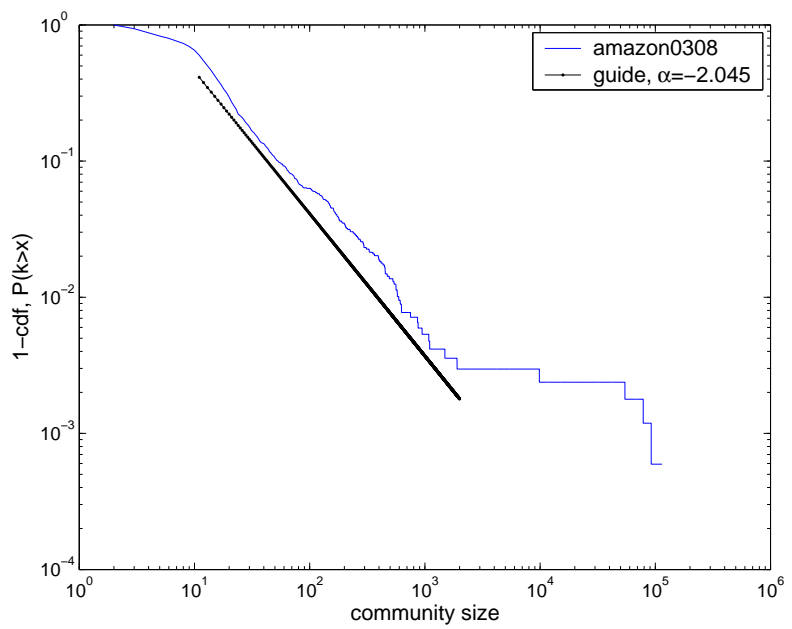

Figure 13: Cumulative distribution of community sizes for the Amazon purchasing network. The partition is derived by greedy modularity optimization. Reprinted figure with permission from Clauset A, Newman MEJ and Moore C, Physical Review E 70, 066111, 2004. Copyright 2004 by the Americal Physical Society.

with the average internal degree of the vertices in the module. The participation ratio says how the edges of the vertex are distributed among the modules. Based on these two indices, Guimerá and Amaral distinguish seven roles for a vertex. These roles seem to be correlated to functions of vertices: in metabolic networks, for instance, vertices sharing many edges with vertices of other modules ("connectors") are often metabolites which are more conserved across species than other metabolites, i.e. they have an evolutionary advantage [12.

\section{Future Directions}

The problem of community detection is truly interdisciplinary. It involves scientists of different disciplines both in the design of algorithms and in their applications. The past years have witnessed huge progresses and novelties in this topic. Many methods have been developed, based on various principles. Their scalability has improved by at least one power in the graph size in just a couple of years. Currently partitions in graphs with up to millions of vertices can be found. From this point of view, the limit is close, and future improvements in this sense are unlikely. Algorithms running in linear time are very quick, but their results are often not very good.

The major breakthrough introduced by the new methods is the possibility of extracting graph partitions with no preliminary knowledge or inputs about 
the community structure of the graph. Most new algorithms do not need to know how many communities there are, a major drawback of computer science approaches: they derive this information from the graph topology itself. Similarly, algorithms of new generation are able to select one or a few meaningful partitions, whereas social science approaches usually produce a whole hierarchy of partitions, which they are unable to discriminate. Especially in the last two years, the quality of the output produced by some algorithms has considerably improved. Realistic aspects of community structure, like overlapping and hierarchical communities, are now often taken into account.

The main question is: is there at present a good method to detect communities in graphs? The answer depends on what is meant by "good". Several algorithms give satisfactory results when they are tested as described in Section [6, in this respect, they can be considered good. However, if examined in more detail, some methods disclose serious limits and biases. For instance, the most popular method used nowadays, modularity optimization, is likely to give problems in the analysis of large graphs. Most algorithms are likely to fail in some limit, still one can derive useful indications from them: from the comparison of partitions derived by different methods one could extract the cores of real communities. The ideal method is one that delivers meaningful partitions and handles overlapping communities and hierarchy, possibly in a short time. No such method exists yet.

Finding a good method for community detection is a crucial endeavour in biology, sociology and computer science. In particular, biologists often rely on the application of clustering techniques to classify their data. Due to the bioinformatics revolution, gene regulatory networks, protein-protein interaction networks, metabolic networks, etc., are now much better known that they used to be in the past and finally susceptible to solid quantitative investigations. Uncovering their modular structure is an open challenge and a necessary step to discover properties of elementary biological constituents and to understand how biological systems work.

\section{References}

[1] Euler L (1736) Solutio problematis ad geometriam situs pertinentis. Commentarii Academiae Petropolitanae, Vol 8, pp 128-140

[2] Bollobás B (1998) Modern Graph Theory. Springer Verlag, New York (USA).

[3] Wasserman S, Faust K (1994) Social Network Analysis: Methods and Applications. Cambridge University Press, Cambridge (UK).

[4] Scott JP (2000) Social Network Analysis. Sage Publications Ltd., London (UK).

[5] Barabási Al, Albert R (2002) Statistical mechanics of complex networks. Reviews of Modern Physics, Vol 74, pp 47-97 
[6] Dorogovtsev SN, Mendes JFF (2003) Evolution of Networks: from biological nets to the Internet and WWW. Oxford University Press, Oxford (UK)

[7] Newman MEJ (2003) The structure and function of complex networks. SIAM Review, Vol 45, pp 167-256

[8] Pastor-Satorras R, Vespignani A (2004) Evolution and structure of the Internet: A statistical physics approach. Cambridge University Press, Cambridge (UK)

[9] Boccaletti S, Latora V, Moreno Y, Chavez M, Hwang DU (2006) Complex Networks: Structure and Dynamics. Physics Reports, Vol 424, pp 175-308

[10] Erdös P, Rényi A (1959) On Random Graphs. Publicationes Mathematicae Debrecen, Vol 6, pp 290-297

[11] Flake GW, Lawrence S, Lee Giles C, Coetzee FM (2002) Self-Organization and Identification of Web Communities. IEEE Computer, Vol 35, No 3, pp $66-71$

[12] Guimerà R, Amaral LAN (2005) Functional cartography of complex metabolic networks. Nature 433, pp 895-900

[13] Palla G, Derényi I, Farkas I, Vicsek T (2005) Uncovering the overlapping community structure of complex networks in nature and society. Nature, Vol 435 , pp $814-818$

[14] Girvan M, Newman MEJ (2002) Community structure in social and biological networks. Proceedings of the National Academy of Science of the USA, Vol 99, No 12, pp 7821-7826

[15] Lusseau D, Newman MEJ (2004) Identifying the role that animals play in their social networks. Proceedings of the Royal Society of London B, Vol 271, pp S477-S481

[16] Pimm SL (1979) The structure of food webs. Theoretical Population Biolology, Vol 16, pp 144-158

[17] Krause AE, Frank KA, Mason DM, Ulanowicz RE, Taylor WW (2003) Compartments exposed in food-web structure. Nature, Vol 426, p 282-285

[18] Granovetter M (1973) The Strength of Weak Ties. American Journal of Sociology, Vol 78, pp 1360-1380

[19] Burt RS (1976) Positions in Networks. Social Forces, Vol 55, No 1, pp 93-122

[20] Freeman LC (1977) A Set of Measures of Centrality Based on Betweenness. Sociometry, Vol 40, No 1, pp 35-41 
[21] Pollner P, Palla G, Vicsek T (2006) Preferential attachment of communities: The same principle, but a higher level. Europhysics Letters, Vol 73, No 3, pp 478-484

[22] Newman MEJ (2004) Detecting community structure in networks. European Physical Journal B, Vol 38, pp 321-330

[23] Danon L, Duch J, Arenas A, Díaz-Guilera A (2007) Community structure identification. Large Scale Structure and Dynamics of Complex Networks: From Information Technology to Finance and Natural Science, Caldarelli G and Vespignani A, Eds., World Scientific, Singapore, pp 93-114

[24] Bron C, Kerbosch J (1973) Finding all cliques on an undirected graph. Communications of ACM, Vol 16, pp 575-577

[25] Radicchi F, Castellano C, Cecconi F, Loreto V, Parisi D (2004) Defining and identifying communities in networks. Proceedings of the National Academy of Science of the USA, Vol 101, No 9, pp 2658-2663

[26] Newman MEJ, Girvan M (2004) Finding and evaluating community structure in networks. Physical Review E, Vol 69, 026113

[27] Arenas A, Fernández A, Fortunato S, Gómez S (2007) Motif-based communities in complex networks. Eprint arXiv:0710.0059 in www.arxiv.org

[28] Reichardt J, Bornholdt S (2006) Statistical mechanics of community detection. Physical Review E, Vol 74, 016110

[29] Massen CP, Doye JPK (2006) Thermodynamics of community structure. Eprint cond-mat/0610077 in www.arxiv.org

[30] Arenas A, Duch J, Fernándes A, Gómez S (2007) Size reduction of complex networks preserving modularity. New Journal of Physics, Vol 9, No 6, pp $176-180$

[31] Guimerà R, Sales-Pardo M, Amaral LAN (2004) Modularity from fluctuations in random graphs and complex networks. Physical Review E, Vol 70, $025101(\mathrm{R})$

[32] Fortunato S, Barthélemy M (2007) Resolution limit in community detection. Proceedings of the National Academy of Science of the USA, Vol 104, No 1, pp 36-41

[33] Gfeller D, Chappelier J-C, De Los Rios P (2005) Finding instabilities in the community structure of complex networks. Physical Review E, Vol 72, 056135

[34] Pothen A (1997) Graph partitioning algorithms with applications to scientific computing. In Parallel Numerical Algorithms, Keyes DE, Sameh A and Venkatakrishnan V, Eds., Kluwer Academic Press, pp 323-368 
[35] Kernighan BW, Lin S (1970) An efficient heuristic procedure for partitioning graphs. The Bell System Technical Journal, Vol 49, pp 291-307

[36] Golub GH, Van Loan CF (1989) Matrix computations. John Hopkins University Press, Baltimore, MD, USA

[37] J. B. MacQueen (1967) Some methods for classification and analysis of multivariate observations. Proceedings of 5-th Berkeley Symposium on Mathematical Statistics and Probability, Berkeley, University of California Press, pp 281-297

[38] Brandes, U (2001) A faster algorithm for betweenness centrality. Journal of Mathematical Sociology, Vol 25, No 2, pp 163-177

[39] Newman MEJ (2005) A measure of betweenness centrality based on random walks. Social Networks, Vol 27, pp 39-54

[40] Tyler JR, Wilkinson DM, Huberman BA (2003) Email as spectroscopy: automated discovery of community structure within organizations. Proceeding of the First International Conference on Communities and Technologies, Huysman M, Wenger E and Wulf V, Eds., Kluwer Academic Press, Amsterdam.

[41] Wilkinson DM, Huberman BA (2004) A method for finding communities of related genes. Proceedings of the National Academy of Science of the USA, Vol 101, Suppl 1, pp 5241-5248

[42] Latora V, Marchiori M (2001) Efficient behavior of small-world networks. Physical Review Letters, Vol 87, 198701

[43] Fortunato S, Latora V, Marchiori M (2004) A method to find community structures based on information centrality. Physical Review E, Vol 70, 056104

[44] Watts D, Strogatz SH (1998) Collective dynamics of "small-world" networks. Nature, Vol 393, pp 440-442

[45] Brandes U, Delling D, Gaertler M, Görke R, Hoefer M, Nikoloski Z, Wagner D (2007) On finding graph clusterings with maximum modularity. Proceedings of the 33rd International Workshop on Graph-Theoretical Concepts in Computer Science (WG'07), Springer Verlag, Berlin-Heidelberg, Germany.

[46] Newman MEJ (2004) Fast algorithm for detecting community structure in networks. Physical Review E, Vol 69, 066133

[47] Clauset A, Newman MEJ, Moore C (2004) Finding community structure in very large networks. Physical Review E, Vol 70, 066111

[48] Danon L, Díaz-Guilera A, Arenas A (2006) The effect of size heterogeneity on community identification in complex networks. Journal of Statistical Mechanics: Theory and Experiment, Issue 11, P11010 
[49] Pujol JM, Béjar J, Delgado J (2006) Clustering algorithm for determining community structure in large networks. Physical Review E, Vol 74, 016107

[50] Kirkpatrick S, Gelatt CD, Vecchi MP (1983) Optimization by simulated annealing. Science, Vol 220, No 4598, pp 671-680

[51] Massen CP, Doye JPK (2005) Identifying communities within energy landscapes. Physical Review E, Vol 71, 046101

[52] Boettcher S, Percus AG (2001) Optimization with extremal dynamics. Physical Review Letters, Vol 86, pp 5211-5214

[53] Duch J, Arenas A (2005) Community detection in complex networks using extremal optimization. Physical Review E, Vol 72, 027104

[54] Newman MEJ (2006) Modularity and community structure in networks. Proceedings of the National Academy of Science of the USA, Vol 103, No. 23, pp 8577-8582

[55] Newman MEJ (2006) Finding community structure in networks using the eigenvectors of matrices. Physical Review E, Vol 74, 036104

[56] Reichardt J, Bornholdt S (2007) Partitioning and modularity of graphs with arbitrary degree distribution. Physical Review E, Vol 76, 015102(R)

[57] Reichardt J, Bornholdt S (2006) When are networks truly modular? Physica D, Vol 224, pp 20-26

[58] Kumpula JM, Saramäki J, Kaski K, Kertész J (2007) Limited resolution in complex network community detection with Potts model approach. European Physical Journal B, Vol 56, pp 41-45

[59] Arenas A, Fernándes A, Gómez S (2007) Multiple resolution of the modular structure of complex networks. Eprint physics/0703218 at www.arxiv.org

[60] Ruan J, Zhang W (2007) Identifying network communities with high resolution. Eprint arXiv: 0704.3759 in www.arxiv.org, to appear in Physical Review E

[61] Kumpula JM, Saramäki J, Kaski K, Kertész J (2007) Limited resolution and multiresolution methods in complex network community detection. Noise and Stochastics in Complex Systems and Finance, Kertész J, Bornholdt S and Mantegna RN, Eds. Proceedings of the SPIE, Vol 6601, pp 660116

[62] Muff S, Rao F, Caflisch A (2005) Local modularity measure for network clusterizations. Physical Review E, Vol 72, 056107

[63] Donetti L, Muñoz MA (2004) Detecting network communities: a new systematic and efficient algorithm. Journal of Statistical Mechanics: Theory and Experiment, P10012 
[64] Capocci A, Servedio VDP, Caldarelli G, Colaiori F (2004) Detecting communities in large networks. Physica A, Vol 352, No 2-4, pp 669-676

[65] Wu F, Huberman BA (2004) Finding communities in linear time: a physics approach. European Physical Journal B, Vol 38, pp 331-338

[66] Eriksen KA, Simonsen I, Maslov S, Sneppen K (2003) Modularity and extreme edges of the Internet. Physical Review Letters, Vol 90, No 14, 148701

[67] Simonsen I, Eriksen KA, Maslov S, Sneppen K (2004) Diffusion on complex networks: a way to probe their large-scale topological structure. Physica A, Vol 336, pp 163-173

[68] Wu FY (1982) The Potts model. Reviews of Modern Physics, Vol 54, pp $235-268$

[69] Blatt M, Wiseman S, Domany E (1996) Superparamagnetic clustering of data. Physical Review Letters, Vol 76, No 18, pp 3251-3254

[70] Reichardt J, Bornholdt S (2004) Detecting fuzzy community structure in complex networks. Physical Review Letters, Vol 93, No 21, 218701

[71] Reichardt J, Bornholdt S (2006) Statistical mechanics of community detection Physical Review E, Vol 74, 016110

[72] Mezard M, Parisi G, Virasoro M (1987) Spin glass theory and beyond. World Scientific Publishing Company, Singapore.

[73] Zhou H (2003) Network landscape from a Brownian particle's perspective. Physical Review E, Vol 67, 041908

[74] Zhou H (2003) Distance, dissimilarity index, and network community structure. Physical Review E, Vol 67, 061901

[75] Zhou H, Lipowsky R (2004) Network Brownian motion: A new method to measure vertex-vertex proximity and to identify communities and subcommunities. Lecture Notes in Computer Science, Vol 3038, pp 1062-1069

[76] Latapy M, Pons P 92005) Computing communities in large networks using random walks. Lecture Notes in Computer Science, Vol 3733, pp 284-293

[77] Arenas A, Díaz-Guilera A, Pérez-Vicente CJ (2006) Synchronization reveals topological scales in complex networks. Physical Review Letters, Vol 96, 114102

[78] Kuramoto Y (1984) Chemical Oscillations, Waves and Turbulence. Springer-Verlag, Berlin, Germany

[79] Arenas A, Díaz-Guilera A (2007) Synchronization and modularity in complex networks. European Physical Journal ST, Vol 143, pp 19-25 
[80] Boccaletti S, Ivanchenko M, Latora V, Pluchino A, Rapisarda A (2007) Detecting complex network modularity by dynamical clustering. Physica Review E, Vol 76, 045102(R)

[81] Pluchino A, Latora V, Rapisarda A (2005) Changing opinions in a changing world: a new perspective in sociophysics. International Journal of Modern Physics C, Vol 16, No 4, pp 505-522

[82] Farkas I, bel D, Palla G, Vicsek T (2007) Weighted network modules. New Journal of Physics, Vol 9, pp 180

[83] Palla G, Farkas IJ, Pollner P, Derényi I, Vicsek T (2007) Directed network modules. New Journal of Physics, Vol 9, pp 186

[84] Palla G, Barabási A-L, Vicsek T (2007) Quantifying social groups evolution. Nature, Vol 446, pp 664-667

[85] van Dongen S (2000) Graph Clustering by Flow Simulation. PhD thesis, University of Utrecht, Netherlands

[86] Newman MEJ, Leicht E (2007) Mixture models and exploratory analysis in networks. Proceedings of the National Academy of Science of the USA, Vol 104, No 23, pp 9564-9569

[87] Bagrow JP, Bollt EM (2005) Local method for detecting communities. Physical Review E, Vol 72, 046108

[88] Clauset A (2005) Finding local community structure in networks. Physical Review E, Vol 72, 026132

[89] Eckmann J.-P, Moses E (2002) Curvature of co-links uncovers hidden thematic layers in the World Wide Web. Proceedings of the National Academy of Science of the USA, Vol 99, No 9, pp 5825-5829

[90] Sales-Pardo M, Guimerá R, Amaral LAN (2007) Extracting the hierarchical organization of complex systems. Eprint arXiv:0705.1679 in www.arxiv.org

[91] Rosvall M, Bergstrom CT (2007) An information-theoretic framework for resolving community structure in complex networks. Proceedings of the National Academy of Science of the USA, Vol 104, No 18, pp 7327-7331

[92] Shannon CE, Weaver V (1949) The Mathematical Theory of Communication. University of Illinois Press, Champaign, USA

[93] Danon L, Díaz-Guilera A, Duch J, Arenas A (2005) Comparing community structure identification. Journal of Statistical Mechanics: Theory and Experiment, P09008

[94] Gustafsson M, Hörnquist M, Lombardi A (2006) Comparison and validation of community structures in complex networks. Physica A, Vol 367, pp 559576 
[95] Zachary WW (1977) An information flow model for conflict and fission in small groups. Journal of Anthropological Research, Vol 33, pp 452-473

[96] Lusseau D (2003) The emergent properties of a dolphin social network. Proceedings of the Royal Society of London B, Vol 270 (Suppl. 2), pp S186188

[97] Guimerá R, Amaral LAN (2005) Cartography of complex networks: modules and universal roles. Journal of Statistical Mechanics: Theory and Experiment, P02001 